\title{
Joint Inflammation and Early Degeneration Induced by High-Force Reaching Are Attenuated by Ibuprofen in an Animal Model of Work-Related Musculoskeletal Disorder
}

\author{
Jeffrey B. Driban, ${ }^{1}$ Ann E. Barr, ${ }^{2}$ Mamta Amin, ${ }^{3}$ Michael R. Sitler, ${ }^{4}$ and Mary F. Barbe ${ }^{3}$ \\ ${ }^{1}$ Division of Rheumatology, Tufts Medical Center, 800 Washington Street, Box 406, Boston, MA 02111, USA \\ ${ }^{2}$ College of Health Professions, Pacific University, 190 SE 8th Avenue, Suite 230, Hillsboro, OR 97123, USA \\ ${ }^{3}$ Department of Anatomy \& Cell Biology, Temple University School of Medicine, 3500 North Broad Street, Philadelphia, PA, \\ 19140, USA \\ ${ }^{4}$ College of Health Professions \& Social Work, Temple University, 3307 North Broad Street, Suite 300, Philadelphia, PA 19140, USA
}

Correspondence should be addressed to Mary F. Barbe, mbarbe@temple.edu

Received 12 September 2010; Accepted 11 December 2010

Academic Editor: Oreste Gualillo

Copyright (C) 2011 Jeffrey B. Driban et al. This is an open access article distributed under the Creative Commons Attribution License, which permits unrestricted use, distribution, and reproduction in any medium, provided the original work is properly cited.

\begin{abstract}
We used our voluntary rat model of reaching and grasping to study the effect of performing a high-repetition and high-force (HRHF) task for 12 weeks on wrist joints. We also studied the effectiveness of ibuprofen, administered in the last 8 weeks, in attenuating HRHF-induced changes in these joints. With HRHF task performance, ED1+ and COX2+ cells were present in subchondral radius, carpal bones and synovium; IL-lalpha and TNF-alpha increased in distal radius/ulna/carpal bones; chondrocytes stained with Terminal deoxynucleotidyl Transferase- (TDT-) mediated dUTP-biotin nick end-labeling (TUNEL) increased in wrist articular cartilages; superficial structural changes (e.g., pannus) and reduced proteoglycan staining were observed in wrist articular cartilages. These changes were not present in normal controls or ibuprofen treated rats, although IL-1alpha was increased in reach limbs of trained controls. HRHF-induced increases in serum C1,2C (a biomarker of collagen I and II degradation), and the ratio of collagen degradation to synthesis (C1,2C/CPII; the latter a biomarker of collage type II synthesis) were also attenuated by ibuprofen. Thus, ibuprofen treatment was effective in attenuating HRHF-induced inflammation and early articular cartilage degeneration.
\end{abstract}

\section{Introduction}

Work-related upper limb disorders in the United States are estimated to cost over $\$ 61.2$ billion annually. In 2006 , the number of occupational injuries involving days away from work due to hand and wrist injuries was 47,020 and 56,250, respectively, while their incidence rates were $10.6 \%$ of $29.5 \%$ total in the upper extremity $[1,2]$. In the United Kingdom, 115,000 new cases of work-related upper limb disorders, also known as repetitive strain injury (RSI), were reported in 2006-2007 compared with 86,000 new cases in 2005-2006, and the number of people reporting problems with RSIs rose from 374,000 to $426,000[3,4]$. Similar increases are reported by the French National Health Insurance Fund-Occupational
Risks, who further break down major work-related musculoskeletal disorders (MSDs) by body region [5]. Their data indicate that the number of new occupational cases resulting in settlement related to peri-articular disorders of the wrist increased $42 \%$ in 2009 , compared to $17 \%$ in the elbow, $6.9 \%$ in the back and $2.2 \%$ in the knee.

Degeneration of joints, including radiocarpal and intracarpal joints, is known to be the result of inter-related traumatic (e.g., a quantitative increase or qualitative aberration in joint loading), inflammatory and metabolic processes [6-8]. Epidemiological studies provide evidence for links between occupational physical activities that involve highly repetitive arm motions and other risk factors for MSDs (e.g., awkward posture, force, duration) and hand and 
wrist disorders, and between occupational physical activities and increased incidence of hand osteoathritis (OA) [9-11]. For example, high incidence of radiographic of hand OA has been identified in middle-aged female dentists and teachers $[10,11]$. In the elderly population, the prevalence of radiographic hand OA can reach $80 \%$ [12]. Several studies report that increasing radiographic severity of hand OA is associated with reduced hand function and pain $[10,13,14]$. Therefore, the impact of hand OA is considerable $[13,14]$.

Several animal models have been used to explore the development of joint degeneration, with the goal of studying the progression of early arthritis and effects of therapeutic interventions. These models lead to a degenerative progression similar to humans, such as from instability-induced joint degeneration, spontaneous degeneration in various strains of mice, use of knockout mice, mice overexpressing certain bone proteins (e.g., overexpression of Runx2 [15$21]$ ). However, several of the initiating factors in these animal models are not representative of the inducing factors in the general population.

We have developed a unique, voluntary rat model of repetitive reaching and grasping that permits the examination of responses of upper limb tissues to cumulative muscular loads [22-32]. This model requires adult rats to voluntarily and repetitively reach for, grasp, and isometrically pull a handle with one forelimb, while the contralateral limb provides postural support, and while both reach rate and force are controlled by food reward criteria. We have shown that a low-demand task (low-repetition and negligible-force; LRNF) induced a low-level and transient inflammatory cytokine tissue response $[25,29]$, while a moderate-demand task (high-repetition and negligible-force; HRNF) induced a moderate inflammatory cytokine response in musculoskeletal tissues, and early pathological woven bone remodeling at distal periosteal-bone sites by 6 weeks of task performance that resolved by 12 weeks [28-30]. The LRNF task regimen consisted of 3.3 reaches $/ \mathrm{min}$ at $<5 \%$ estimated maximum pulling force $(<9.85 \mathrm{~g})$, while the HRNF task regimen consisted of the same force requirements but 8 reaches $/ \mathrm{min}$. Thus, we observed a loading-level dependent inflammatory cytokine response with these two task regimens. In terms of pathological bone remodeling, we observed periosteal hyperplasia, particularly at sites of tendon and muscle attachments to the radius and ulna, and increased cellularity (increased osteoclasts and their progenitor cells, and osteoblasts) in distal cortical bones of the radius and ulna, as well as disruption of laminar bone organization in the HRNF bones. The cellular changes peaked between 4 and 6 weeks but returned to normal by 12 weeks of performance of the HRNF task. Also, by 12 weeks, HRNF task-induced disorganized collagen fibers of the periosteum had begun to regain their closely packed parallel alignment, and lamellar organization was more similar to control bone [30]. These latter findings are indicative of bone adaptation to the cumulative loading of this moderate demand task.

In contrast, bone adaptation was not evident with continued performance of higher-demand tasks (e.g., moderate repetition with high-force (MRHF) or high-repetition with high-force (HRHF)), tasks requiring $60 \%$ maximum isometric pulling force (an average of $118 \mathrm{~g}$ ), for 2 hours/day and 3 days/week [23, 32]. Instead of resolution of the inflammatory response, we observed continued (chronic) inflammatory responses in nerve and musculoskeletal tissues and increased pathological bone remodeling that was again dependent on the level of the task, with the greatest changes observed with the HRHF task [23, 26, 31, 32]. With 12 weeks of performance of an HRHF task, we observed significantly decreased height of the distal radial and ulnar epiphyseal plates, decreased cortical bone thickness, reduction in a bone matrix repair molecule (periostin like factor), and increased Trap5b (a serum marker of osteoclast activity and bone resorption [31,32]). Thus, we have observed the induction of pathological bone changes with higher-demand tasks in our animal model, a task that incorporates both a quantitative increase in bone loading and cumulative loading. However, the influence of the high-demand tasks on joint tissues has not been assessed in our model, nor has the inflammatory response in bones of $\mathrm{HRHF}$ rats past 6 weeks of task performance. Continued exploration of the inflammatory response using this rat model in which repetition and force requirements can be varied with operant behavioral training, and in which inflammation (or at least aspects of it) is attenuated with anti-inflammatory drugs, is needed to fully understand the links between biomechanical factors and inflammation.

One purpose of this study was to evaluate the potential of a voluntary HRHF rat model to induce inflammation and articular cartilage degeneration in radiocarpal and intracarpal joints. We hypothesized that performance of an HRHF forearm/hand intensive task would induce increased inflammatory cytokines and the onset of degenerative changes in wrist joint structures. A second purpose was to determine the effectiveness of oral ibuprofen, a nonselective cyclooxygenase inhibitor, on these outcomes, since oral ibuprofen is a commonly used nonselective nonsteroidal anti-inflammatory drug (NSAID) for the treatment of OA [33-37]. We hypothesized that a steady dose of ibuprofen would attenuate the increase in inflammatory cytokines that we have previously observed in the distal radius and ulna and carpal bones in our model, albeit when induced by lower-demand tasks $[25,29]$. We further hypothesized that use of ibuprofen would contribute to an attenuation of any observed radiocarpal and intracarpal cartilage degenerative changes despite continued loading of the joints from continued task performance during the ibuprofen treatment, although the ability of ibuprofen to attenuate articular cartilage degenerative changes is controversial and still under investigation [35-37].

\section{Materials and Methods}

2.1. Animals. Animal care and use were monitored by the University Animal Care and Use Committee to assure compliance with the provisions of Federal and National Institute of Health regulations. Eighty-seven adult female SpragueDawley rats (3.5 months of age at onset of experiments) were obtained from ACE (Boyertown, PA). They were housed in separate cages in a 12-hour light/dark cycle. Rat weights 
were measured weekly throughout the study. All trained and task animals were food restricted for no longer than 1 week to no less than $80 \%$ of full body weight during the first week of the initial training period when they first began learning the task. However, during the remaining 5 weeks of training, they were maintained at $\pm 5 \%$ of agematched normal controls with rat chow daily to supplement the food pellets used for food reward ( $45 \mathrm{mg}$ purified formula food pellets; Bioserve, Frenchtown, NJ). All rats were given free access to water throughout the experiment. Trained only control rats matched experimental animals by age and weight, and were sacrificed at matched time points. Normal controls with free access to food were matched to experimental animals by age and weight (within $\pm 5 \%$ after the first week), and were sacrificed at matched time points.

Eighty-seven animals were randomly assigned to one of six groups. There were two experimental groups: rats trained to perform an HRHF task without ibuprofen for 12 weeks (HRHF, $n=18$ ), and HRHF rats that received ibuprofen treatment (HRHF + IBU, $n=15)$. There were four control groups: trained controls (TR, $n=14$ ), TR that received ibuprofen treatment (TR + IBU, $n=11)$, untrained normal controls (NC, $n=19$ ), and NC that received ibuprofen treatment $(\mathrm{NC}+\mathrm{IBU}, n=10)$.

2.2. Behavioral Apparatus, Training Procedures, and Task Performance. The behavioral apparatuses used were as described and depicted previously [26, 38]. Briefly, force apparatuses custom designed by Ann Barr and Custom Medical Research Equipment (Glendora, NJ, USA) were used that were integrated into a computerized operant behavioral training system (Med Associates, Georgia, VT, USA with Force Lever software, version 1.03.02, Med Associates). A portal was located in the chamber walls at shoulder height $(3.5 \mathrm{~cm})$, requiring shoulder elevation and elbow extension for the animal to reach through the portal to a customdesigned force handle attached to a force transducer located $1.5 \mathrm{~cm}$ away from the portal entrance, outside the chamber wall. An auditory indicator cued the animals to reach. HRHF rats had to grasp the force handle and exert an isometric pull toward the chamber wall for at least 50 milliseconds with a graded force effort of $60 \pm 5 \%$ average maximum pulling force (MPF; $108.35 \mathrm{~g}$ to $128.05 \mathrm{~g}$ range of pulling force), as determined on the last day of training. If these force and time criteria were met within a 5-second cueing period, an indicator light turned on and a $45 \mathrm{mg}$ purified formula food pellet (banana flavored; Bioserve, NJ, USA) was dispensed into a trough located at floor height of the chamber. Animals were allowed to use their preferred limb to reach, which was recorded. The contralateral limb was used as a postural support limb throughout the reach and pull task.

Sprague-Dawley rats were 3.5 month old at the onset of the experiment. Fifty-eight rats were trained to perform a repetitive handle-pulling task with food reward using standard operant conditioning procedures during a 6-week training period. During this training period, rats learned the HRHF task in the operant test chamber until they could reach into the tube dispenser with no specified reach rate at $60 \pm 5 \%$ maximum pulling force for $10 \mathrm{~min} /$ day. Once the animals were able to perform the task consistently (usually after 6 weeks), rats were randomly divided into trained only control (TR) and HRHF groups. Then the HRHF animals $(n=33)$ began the task regimen at a rate of 12 reaches $/ \mathrm{min}$ at $60 \pm 5 \% \mathrm{MPF}$, for $2 \mathrm{~h} /$ day, 3 days/week for 12 weeks. The daily task was divided into four, 0.5 -hour training sessions separated by 1.5 hour. The TR rats $(n=25)$ did not perform beyond this initial training period.

2.3. Ibuprofen Treatment. At the end of the 4th week of task performance, subcohorts of the above animals were administered ibuprofen (Children's Motrin Grape Flavored, Johnson \& Johnson) in drinking water daily $(45 \mathrm{mg} / \mathrm{kg}$ body weight): $\mathrm{NC}+\mathrm{IBU}(n=10)$, TR $+\mathrm{IBU}(n=11)$ and HRHF + IBU $(n=15)$. HRHF+IBU animals continued to perform the HRHF task regimen with ibuprofen treatment for the remainder of the 12-week task period (i.e., an 8week course of ibuprofen treatment). The dose used was lower than the maximum limit for gastrointestinal toxicity in rats, yet has been shown to be effective in reducing chronic inflammation [39]. The amount of medicated water consumed/day was tracked for each animal by measuring the difference between the initial and final volume of suspended solution daily. Serum levels of ibuprofen were confirmed using National Medical Services (Willow Grove, PA). Based on these assessments, the average weekly ibuprofen dose was similar in all groups $(48.8 \pm 6.3 \mathrm{mg} / \mathrm{kg}$ body weight), with no significant differences in ibuprofen dose administered or serum levels of ibuprofen between the treated groups.

2.4. Wrist Measurement. Wrist girth was measured in millimeters for all animals using an electronic caliper (Mitutoyo Vernier Pointed Jay Caliper, model 536-121) immediately after anesthesia (see the following) and before euthanasia.

2.5. Tissue Collection for Cytokine Analysis. Following euthanasia by sodium pentobarbital (Nembutol, $120 \mathrm{mg} / \mathrm{kg}$ body weight), wrist joints (distal radius and ulna metaphysis and epiphysis and articular cartilage, and the first row of carpal bones) were collected from subcohorts of animals: NC $(n=7)$ and NC + IBU $(n=6)$, TR $(n=6), \mathrm{TR}+\mathrm{IBU}$ $(n=5)$, HRHF $(n=6)$, and HRHF $+\operatorname{IBU}(n=6)$. Elbow joints (proximal radius and ulna, and distal humerus) were also collected. Tissues were flash-frozen, homogenized, and assessed for interleukin (IL)- $1 \alpha$, IL- $1 \beta$, tumor necrosis factor (TNF) $\alpha$, IL-6 and IL-10, using commercially available ELISA kits (Bio-Source, Invitrogen Life Sciences, CA) as described previously [29]. The sensitivity of the assays was $<3 \mathrm{pg} / \mathrm{mL}$ for IL- $1 \alpha$ and IL- $1 \beta,<0.7 \mathrm{pg} / \mathrm{mL}$ for TNF- $\alpha,<7 \mathrm{pg} / \mathrm{mL}$ for IL6 , and $<5 \mathrm{pg} / \mathrm{mL}$ for IL-10. Each sample was run in duplicate. ELISA assay data (pg cytokine protein) were normalized to $\mu \mathrm{g}$ total protein, which was determined using a bicinchoninic acid (BCA) protein assay kit.

2.6. Histopathological Grading System for Degenerative Changes. Following euthanasia by sodium pentobarbital (Nembutol, $120 \mathrm{mg} / \mathrm{kg}$ body weight), subcohorts of animals were perfused transcardially with $4 \%$ paraformaldehyde in $0.1 \mathrm{M}$ $\mathrm{PO}_{4}$ buffer ( $\left.\mathrm{pH} 7.4\right)$ : NC $(n=8), \mathrm{NC}+\mathrm{IBU}(n=5)$, 
TR $(n=5), \mathrm{TR}+\mathrm{IBU}(n=6), \operatorname{HRHF}(n=9)$, and HRHF + IBU $(n=6)$. Bilateral radius and ulna, with attached carpal bones and ligaments were collected, decalcified, paraffin embedded and sectioned into $5 \mu \mathrm{m}$ longitudinal sections as described previously [30]. Morphological changes in radial articular cartilage were assessed using these paraffin embedded sections after staining with Safranin $\mathrm{O}$ and fast green. A modified Mankin scoring system was used, which was derived from three subscores: (1) structure, (2) cellular abnormalities, and (3) matrix staining, as described previously [17, 40-42]. Within each subscore there was a range of possible values. Within the structure subscore, there were 7 possible scores: $0=$ normal structure, $1=$ irregular surface (including fissures into the radial layer), 2 = pannus, 3 = superficial cartilage layers absent, $4=$ slight disorganization (cellular rows absent, some small superficial clusters), $5=$ fissures into calcified cartilage layer, and 6 disorganization $\geq 25 \%$ (chaotic structure, clusters, osteoclast activity). The cellular abnormalities subscore had 4 grades: $0=$ normal, 1 = hypercellularity (including small superficial clusters), 2 = clusters, and 3 = hypocellularity. The matrix staining subscore had 5 grades: $0=$ normal or slight reduction in staining, 1 = staining reduced in radial layer, 2 = reduced in interterritorial layer, 3 = only present in pericellular matrix, and $4=$ staining absent. The scoring system was supplemented with half points. Intratester reliability in scoring was assessed: Zone $1 \operatorname{ICC}(3,1)=0.91$, Zone $2 \operatorname{ICC}(3,1)$ $=0.85$, Zone $3 \operatorname{ICC}(3,1)=0.66$, and Zone $4 \operatorname{ICC}(3,1)=$ 0.76 . Additional sections stained with hematoxylin and eosin (H\&E) were also used to evaluate articular cartilage structure and cellular subscores. These changes were assessed in a blinded manner by one rater; a second blinded investigator also evaluated $30 \%$ of the joints to verify the appropriate score. Each radiocarpal joint was assessed in four zones, progressing from the radial margin (zone 1) to the ulnar margin (zone 4) of the distal radius articular cartilage (see Figure 3(a)). If a joint had a questionable appearance (e.g., tears or folds produced during slide preparation), adjacent sections were evaluated. Changes in Safranin $\mathrm{O}$ and fast green in the epiphyseal plate were also examined qualitatively.

Also, the reach limb's distal forelimb bones of nine additional rats (NC, $n=3$; TC, $n=3$; HRHF, $n=3$; and HRHF + IBU, $n=3$ ) did not undergo decalcification, but were dehydrated with ethylene glycol monoethyl (Fisher, Fair Lawn, NJ, USA), cleared in methyl salicylate (J.T. Baker, Phillipsburg, NJ), and embedded in methyl methacrylate with 15\% dibutyl phthalate (Fisher Scientific, Pittsburgh, PA). Bones were cut into $5 \mu \mathrm{m}$ longitudinal sections using a diamond saw. These sections were stained with Safranin $\mathrm{O}$ and fast green for qualitative assessment purposes and for confirmation of results from decalcified, paraffin embedded sections.

2.7. Immunohistochemistry and Histochemistry. Immunohistochemistry was also performed on the paraffin-embedded bone sectioned to qualitatively assess the presence of cyclooxygenase 2 (COX 2), ED1 (a marker of activated macrophages, osteoclasts, and their progenitors), and inflammatory cytokine (IL- $1 \alpha$, TNF- $\alpha$, and IL-10) stained cells in radiocarpal articular cartilage, bone, synovium, and ligaments. Bones were prepared and immunostained for ED1 and inflammatory cytokines using antibodies (Millipore, Billerica, MA) and methods described previously [23, 30, 32]. Sections for single-labeling or fluorescence doublelabeling were immunostained with anti-COX2 (sc-1745; Santa Cruz Biotechnology Inc, Santa Cruz, CA) at $1: 300$ dilution in phosphate buffer using similar methods as previously described $[29,38]$.

Terminal deoxynucleotidyl Transferase- (TDT-) mediated dUTP-biotin nick end-labeling (TUNEL) was performed in adjacent paraffin-embedded sections using a TACS TdT kit according to manufacturer's directions (catalog number TA4625, R\&D Systems, Inc). Briefly, after decalcification, sections were washed in PBS, treated with Proteinase $\mathrm{K}$ solution (in kit) for 15 minutes, and washed with $\mathrm{dH}_{2} \mathrm{O}$, immersed in quenching solution for 5 minutes, washed in PBS, immersed in $1 \mathrm{X}$ TdT solution for 5 minutes, incubated with Labeling Reaction Mix for 1 hour at $37^{\circ} \mathrm{C}$, immersed in Stop Buffer for 5 minutes at room temperature, washed with $\mathrm{dH}_{2} \mathrm{O}$ and then PBS before treating with $0.3 \% \mathrm{H}_{2} \mathrm{O}_{2}$ in PBS for 30 minutes before washing again. Sections were then reacted with streptavidin-HRP for $10 \mathrm{~min}$ at $37^{\circ} \mathrm{C}$, washed with PBS, reacted with DAB solution for 5 minutes, washed with $\mathrm{dH}_{2} \mathrm{O}$, dehydrated through a series of increasing alcohol solutions and then xylene before coverslipping with DPX mounting media. Sections were not counterstained. Staining controls included a positive control slide (stomach) in which TUNEL staining should be and was present, an unlabeled control slide that showed no brown staining, a TACS-Nuclease-treated control slide (which showed only pale brown staining in most cells), and sections of distal radius/ulna/carpal bones from normal controls rats.

2.8. Collection of Serum and Measurement of C1, 2C, and CPII. Following euthanasia (which occurred at $18 \mathrm{hrs}$ after completion of the final task session), blood was collected from $\mathrm{NC}(n=11), \mathrm{NC}+\mathrm{IBU}=6, \mathrm{TR}(n=9), \mathrm{TR}+\mathrm{IBU}(n=$ $6), \operatorname{HRHF}(n=13)$ and HRHF + IBU rats $(n=6)$, by cardiac puncture using a 23-gauge needle into plain plastic $15 \mathrm{~mL}$ tubes, placed on ice for $30 \mathrm{~min}$, and centrifuged at $1,000 \mathrm{~g}$ for $20 \mathrm{~min}$ at $4^{\circ} \mathrm{C}$. Serum was then collected, flash-frozen, and stored at $-80^{\circ} \mathrm{C}$ until analyzed. ELISA was used to measure serum concentrations of $\mathrm{C} 1,2 \mathrm{C}$, also known as COL2 3/4 C short (IBEX Technologies, Inc., Montreal, Quebec). This assay measures types I and II collagen degradation using a specific polyclonal antibody to their primary cleavage sites; this antibody recognizes $\alpha$-chain fragments produced by collagenase cleavage (by matrix metalloproteinase- (MMP) 1, MMP-8, MMP-13) of type II collagen. ELISA was also used to measure serum concentrations of CPII, a collagen type II synthesis biomarker that is also known as procollagen II C-propeptide (IBEX Technologies, Inc). This assay measures intact C-propeptide using a specific polyclonal antibody to the type II collagen carboxy propeptide cleaved from type II procollagen following the release of newly synthesized procollagen into the matrix. Data for C1, 2C are presented as $\mathrm{ng} / \mathrm{ml}$ serum. Data for CPII are presented as part of the ratio of collagen degradation to synthesis markers ( $\mathrm{C} 1,2 \mathrm{C} / \mathrm{CPII})$. 
2.9. Statistical Analyses. All data were analyzed with descriptive and interferential statistics using Prism Graph Pad. Statistical significance was based on a $P \leq .05$. All measures of variance are reported as standard error of the mean. Wrist girth was assayed using one-way ANOVA. ELISA data were analyzed using a two-way ANOVA with the factors group and limb (reach and support limbs). Each cytokine was analyzed separately within a $6 \times 2$ ANOVA. Significant findings were assessed with the Bonferroni method of post hoc testing, with adjusted $P$ values, in which data from all groups and limbs, per cytokine, were compared to each other (the adjusted $P$ value for significance was $\leq .002$ ). For the histological scoring data, total scores for each zone were initially analyzed using paired-sample $t$-tests with Bonferroni corrections to assess differences between the four zones. We found that all zones of the articular cartilage were significantly different from control levels except for zones 1 and 4 . Because these two zones were on the margins of the articular cartilage and may represent a transitional region of articular cartilage, only zones 2 and 3 were analyzed further. Thus, a 6 (group) $\times 2(\operatorname{limb}) \times 2$ (zones 2 and 3$)$ ANOVA with zone treated as a within subjects variable was performed to assess differences in histopathological articular cartilage scores between groups and limbs. Limbs were found to not significantly differ from each other; therefore, their data were combined to assess differences using a two-way ANOVA with the factors group and zone (zones 2 and 3). Significant findings for averaged total scores and individual subscores were assessed with appropriate follow-up one-way ANOVAs. Significant findings were then assessed with the Bonferroni method of post hoc testing, with adjusted $\mathrm{p}$ values, in which data from all groups were compared to each other. A one-way ANOVA was performed to assess differences between groups in $\mathrm{C} 1$, 2C serum concentrations and the $\mathrm{C} 1,2 \mathrm{C} / \mathrm{CPII}$ ratio, each followed by the Bonferroni corrected post hoc test method.

\section{Results}

3.1. Wrist Girth. Wrist girth was assayed to determine if there was swelling at this joint as a consequence of task performance. No changes from NC levels were found $(P=$ $.53)$. For example, the wrist girth in HRHF was $6.30 \pm$ $0.09 \mathrm{~mm}$ (mean $\pm \mathrm{SEM})$ compared to $6.18 \pm 0.16 \mathrm{~mm}$ in NC.

\subsection{Increase of Cox 2 and ED1+Cells in Wrist Joint Articular} Cartilage, Synovium and Ligaments. We initially examined $\mathrm{NC}$ and HRHF distal ulna, radiocarpal joint and carpal bones for increased immunoexpression of COX 2. As shown in Figure 1, compared to NC (Figures 1(a) and 1(b)), we found increased COX2 immunopositive $(+)$ chondrocytes in the distal radius articular cartilage and adjacent synovium (Figures 1(c) and 1(d)), distal ulna articular cartilage and carpal bone articular cartilages (Figure 1(e)) in HRHF rats. We also observed increased COX2+ cells in the radiocarpal (Figures 1(e)s and 1(f)) and intracarpal ligaments in HRHF rats compared to NC (NC not shown). Many of the COX2+ cells in the synovium were ED1+ macrophages (Figures $1(\mathrm{~g})-1(\mathrm{i})$ ). Therefore, NC, TR, TR
+ IBU, HRHF, and HRHF + IBU wrist joints were also evaluated for the presence of ED1+ cells (a marker of macrophages, osteoclasts and their progenitors). In HRHF reach limbs, ED1+ mononucleated cells were identified in the subchondral bone adjacent to articular cartilage in the distal radius (Figure 1(k)). Many ED1+ mononucleated cells were also observed in subchondral regions of carpal bones (Figure 1(1), arrows). NC lacked ED1+ cells in subchondral regions of radial and carpal bones (Figure $1(j)$ ), as did TR and TR + IBU rats (data not shown). A small number of ED1+ cells were observed in the same areas in the HRHF + IBU rats, however there were no significant differences between the number of number of ED1+ cells in HRHF + IBU and NC rats $(P>.05$ both before and after the Bonferroni adjustment of the $P$ value; data not shown). This increase in COX2+ and ED1+ cells in HRHF wrist joint structures, increases that were attenuated by the ibuprofen treatment, further justified the use of ibuprofen, an antiinflammatory drug, as an intervention in this study.

\subsection{Increased Inflammatory Cytokines in Distal Radius, Ulna} and Carpal Bones. Since we have previously identified an increase of several proinflammatory cytokines (e.g., TNF$\alpha$ and IL- $1 \alpha)$ in musculoskeletal tissues in response to the HRHF task when performed for 6 weeks, and the effectiveness of an anti-TNF- $\alpha$ drug in attenuating their increases [31], we extended that investigation in this study to examine their production at 12 weeks, as well as the effectiveness of ibuprofen in attenuating this potential increase since ibuprofen is a commonly used NSAID. Biochemical analysis of joint inflammation was assessed in homogenized wrist joints using ELISA. IL- $1 \alpha$ levels were highest in the distal forelimb bones of HRHF reach limbs, compared to NC, NC + IBU, TR, TR + IBU and HRHF + IBU $(P<.01$ each; Figure $2(a))$, and in the HRHF reach limb compared to the support limb $(P<.05$; Figure 2(a)). It was also higher in the TR reach limbs than in NC $(P<.01)$ and NC + IBU $(P<.05)$. IL- $1 \alpha$ levels were not increased in the support limbs of TR rats compared to NC due to the use of Bonferroni corrections and adjusted $P$ values; however, we should note here that the 2-way ANOVA for this cytokine showed no significant differences between limbs $(P=.57)$ including in the TR rats.

TNF- $\alpha$ levels were highest in both the HRHF reach and support limbs compared to all other groups $(P<.01$ each; Figure 2(b)). IL-10 levels were highest in TR distal forelimb bones, bilaterally, compared to NC, NC + IBU, and HRHF + IBU $(P<.01$ each, Figure $2(\mathrm{c}))$, and in the TR reach limb compared to TR + IBU and HRHF reach limbs $(P<.05$ each; Figure 2(c)). IL-10 was also elevated in TR + IBU reach limb compared to NC $(P<.05)$ and in the TR-IBU limbs, bilaterally, compared to HRHF + IBU $(P<.05$ each $)$, and in HRHF compared to NC + IBU limbs $(P<.01$ for reach limb, $P<.05$ for support limb) (Figure 2(c)). IL-6 levels showed significant differences by group $(P=.005)$, with significant post hoc findings of decreased IL- 6 in TR, bilaterally, and in TR + IBU and HRHF + IBU reach limbs, compared to NC + IBU $(P<.05$ each; Figure $2(e))$. IL- $1 \beta$ levels also showed significant differences by group $(P=.03)$, but no significant post hoc findings (Figure $2(\mathrm{~d})$ ). Immunohistochemistry was 


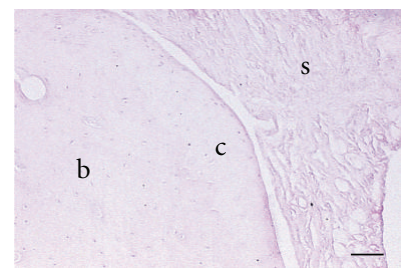

(a)

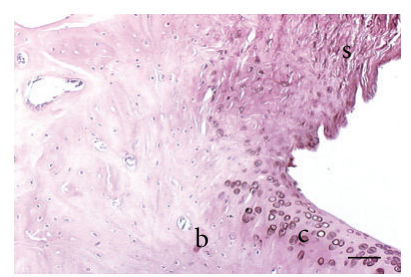

(c)

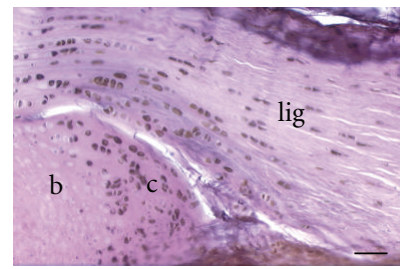

(e)

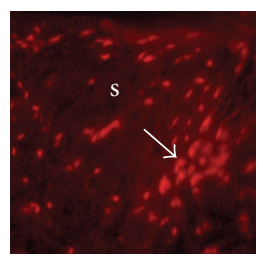

(g)

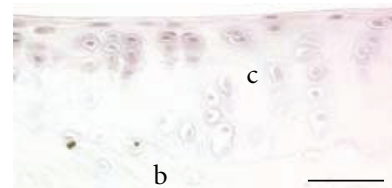

(j)

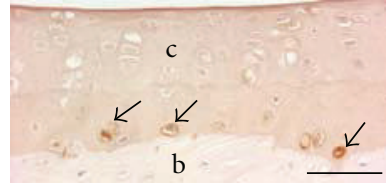

(k)

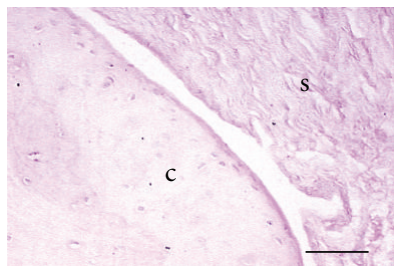

(b)

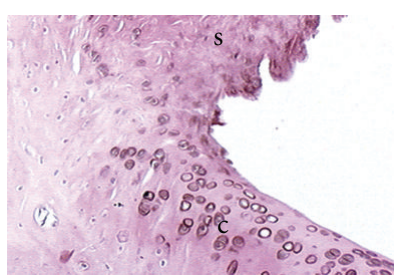

(d)

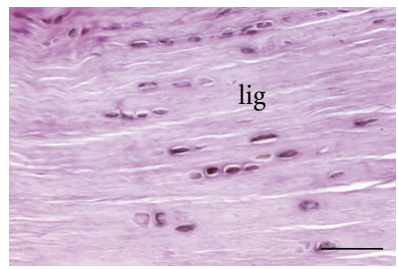

(f)

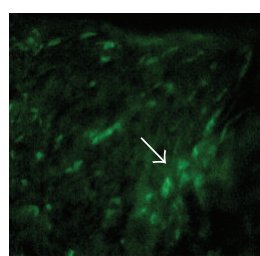

(h)

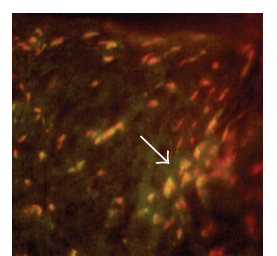

(i)

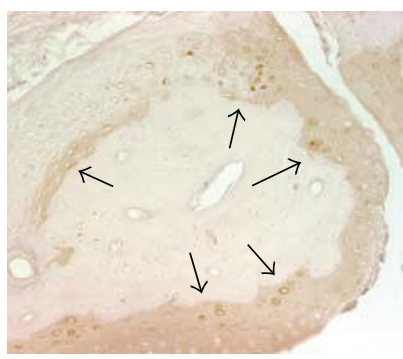

(1)

FIGURE 1: Immunohistological staining cyclooxygenase 2 (COX 2) and ED1 in HRHF distal radiocarpal articular cartilage, synovium and ligaments of longitudinally sections of paraffin embedded bones. ED1 detects a $90 \mathrm{kDa}$ lysosomal membrane glycoprotein in osteoclasts, phagocytic macrophages and their progenitors. (a)-(f) COX 2 staining was visualized using DAB intensified with cobalt (black signal); light hematoxylin and eosin ((h) and (e)) staining was used as a counterstain. (a) No COX 2 immunostaining was observed in distal radial articular cartilage and synovium of a normal control (NC). (b) Higher-power image of same section as shown in (a). (c) COX2+ cells are present in the articular cartilage and synovium of a 12-week high-repetition high-force (HRHF) rat's distal radius. (d) Higher-power image of same section as shown in (c). (e) COX2+ cells are present in the distal radial articular cartilage and radiocarpal ligament of a 12 week HRHF rat. (f) Higher-power image of same section as shown in (e). (g)-(i) Several COX2+ cells are present in the radiocarpal synovium of an HRHF 12 rat (red cells). (g) Same section double labeled for ED1 (green cells, (h)). (i) Merged photo. Arrow indicates a group of COX2+/ED1+ double labeled cells. (j) ED1+ cells (brown DAB signal) were absent in distal radius articular cartilage of an NC rat. Section is lightly counterstained with hematoxylin. (k) Mononucleated ED1+ cells (arrows, presumably osteoclast progenitor cells) were observed in the subchondral bone of the distal radius of an HRHF rat. (l) ED1+ cells were also present in HRHF carpal bones. Scale bars $=50 \mu \mathrm{m}$. b: bone, c: articular cartilage, lig: ligament, s: synovium. 


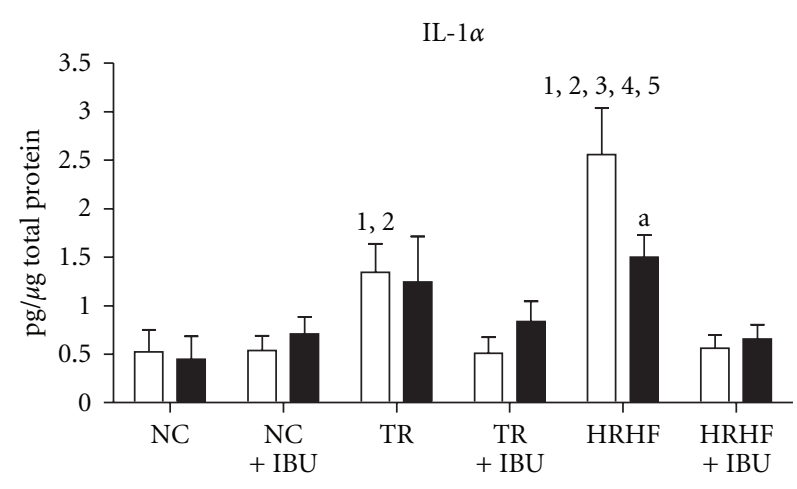

(a)

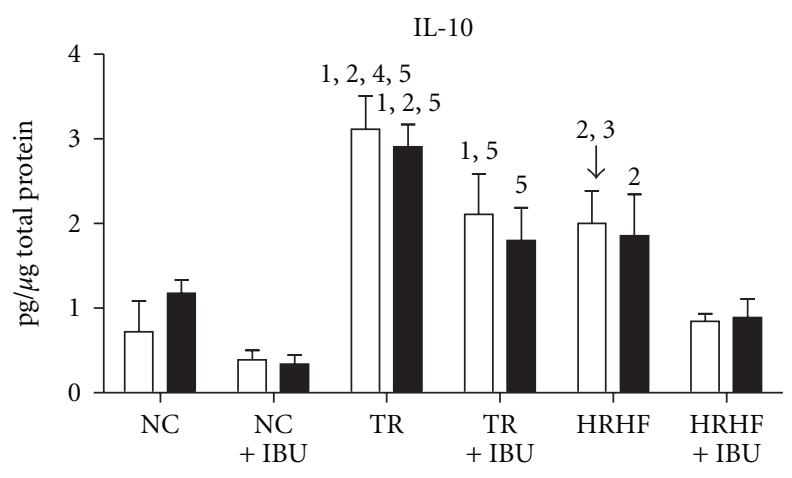

(c)

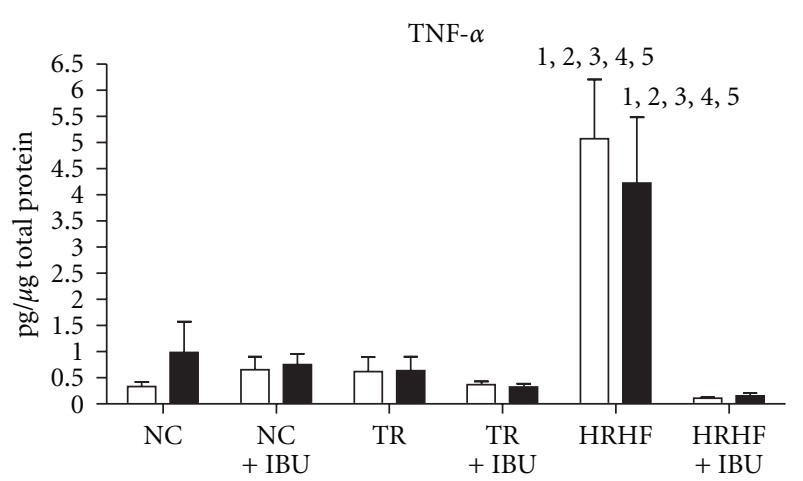

(b)

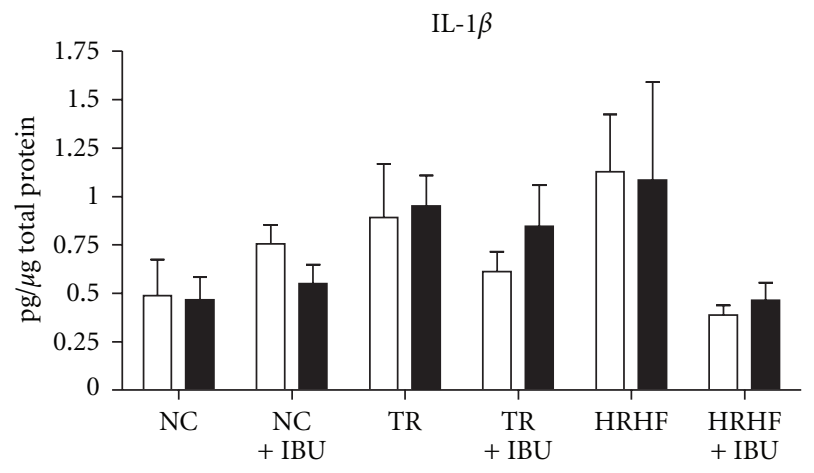

(d)

IL-6

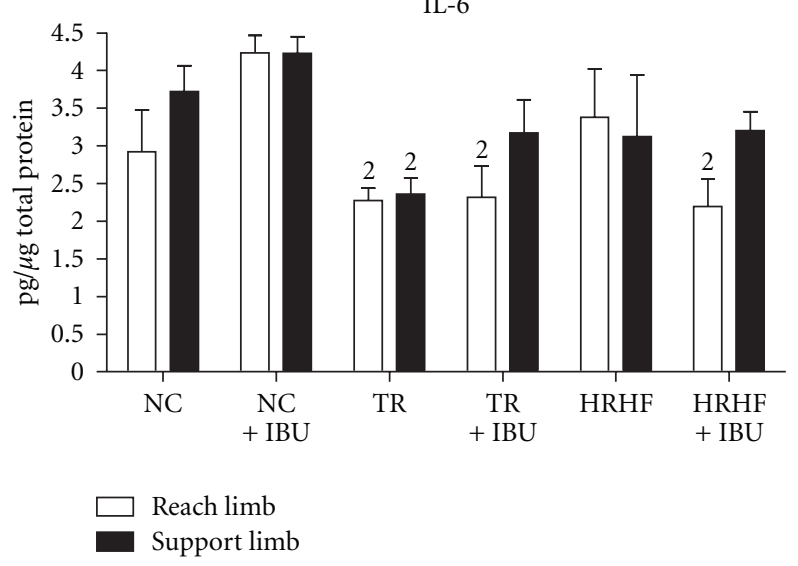

(e)

FIGURE 2: Cytokine concentrations in reach and support wrist joint (distal most radius, distal ulna, first row of carpal bones, and associated joint regions), tested using ELISA. Levels of (a) Interleukin (IL)- $1 \alpha$, (b) tumor necrosis factor (TNF)- $\alpha$, (c) IL-10, (d) IL-1 $\beta$, and (e) IL-6 are shown for NC (normal control), NC + IBU (normal controls receiving ibuprofen for 8 weeks), TR (trained controls), TR + IBU (trained controls receiving ibuprofen treatment in final 8 weeks), HRHF (rats performing high-repetition high-force task for 12 weeks), and HRHF + IBU (HRHF rats receiving ibuprofen treatment in final 8 weeks). Abbreviations: $1=P<.05$ compared to NC, $2=P<.05$ compared to $\mathrm{NC}+\mathrm{IBU}, 3=P<.05$ compared to TR; $4=P<.05$ compared to TR + IBU; $5=P<.05$ compared to HRHF + IBU; a support limb is significantly different from reach limb, $P<.05$.

used to determine that IL- $1 \alpha$ and TNF- $\alpha$ immunoreaction product was present in synovial cells and chondrocytes of the articular cartilage (data not shown). We also observed using double-labeling immunohistochemistry that most of the small ED1+ mononucleated cells were also immunoreactive for IL- $1 \alpha$ and TNF- $\alpha$, matching results of several previous studies in our lab showing expression of inflammatory cytokines by ED1 immunoreactive cells in musculoskeletal and nerve tissues $[23,28,38]$.

To determine if the increases in cytokines were widespread throughout the forelimb, we also examined the same 5 cytokines in reach limb elbow joints. None were 


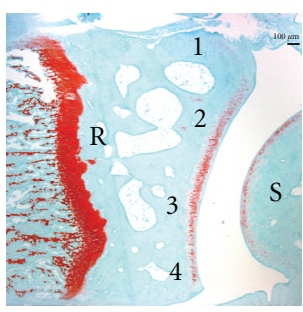

(a)

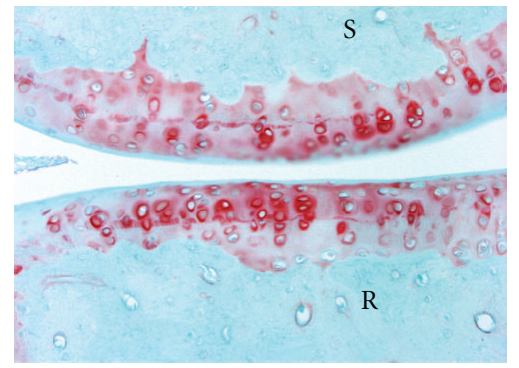

(e)

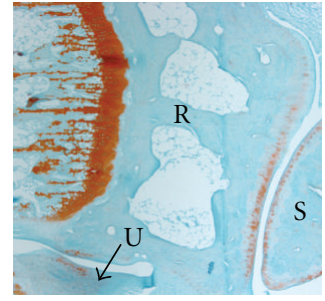

(b)

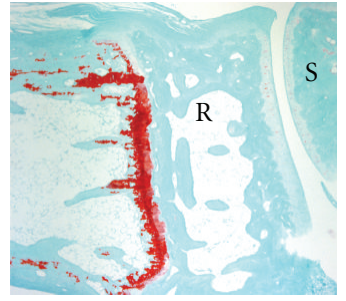

(c)
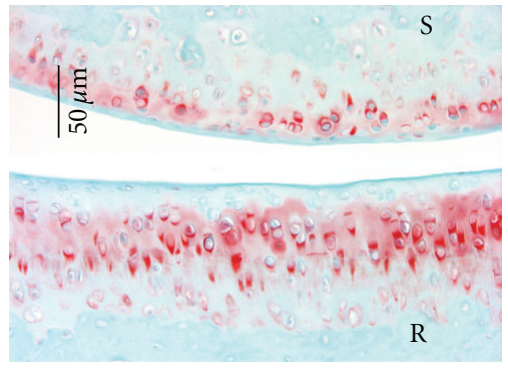

(d)

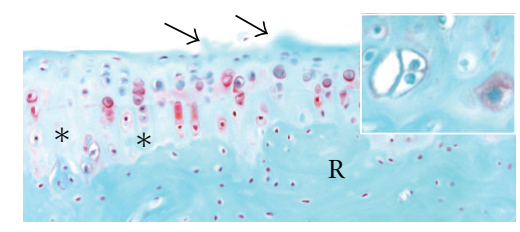

(f)

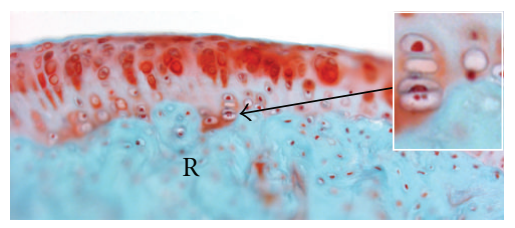

(g)

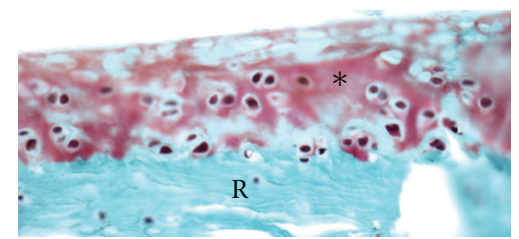

(h)
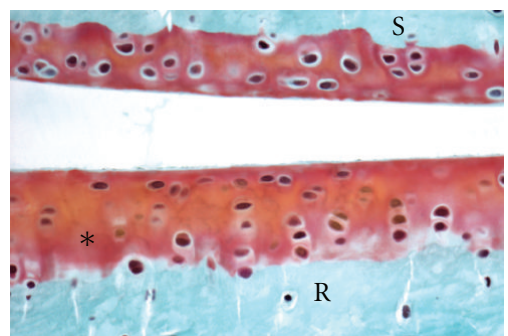

(i)

FIgURE 3: Distal radii and carpal bones stained with safranin $\mathrm{O}$ and fast green. (a)-(g) are longitudinal sections of paraffin embedded radius, ulna and carpal bones. (a)-(c) Low-power micrographs showing distal radius, scaphoid bones and articular cartilage in (a) NC (normal control), (b) TR (trained control), and (c) HRHF rat (performed the high-repetition high-force task for 12 weeks). (a) also indicates the 4 zones of the articular cartilage assessed in the radius. (d)-(g) Higher-power images showing the distal radii articular cartilage of (d) untreated TR, (e) TR + IBU (trained controls receiving ibuprofen treatment in final 8 weeks), (f) HRHF, and (g) HRHF + IBU rats. (f) demonstrates an HRHF animal with structural changes (surface pannus, arrows), cellular changes (hypocellularity in areas indicated with *), and dramatically reduced proteoglycan staining in the articular cartilage (red-pink safranin O staining). The inset in (f) is a higher-power micrograph from another HRHF rat showing chondrocytes that appear to be proliferating. The inset in (g) shows a higher-power micrograph from area indicated with arrow that also contains proliferating chondrocytes. (h)-(i) Loss of safranin O staining in HRHF articular cartilage was verified in plastic embedded bones that were also cut longitudinally. Radii and scaphoid articular cartilages of (h) HRHF and (i) HRHF + IBU are shown. Scale bars $=50 \mu \mathrm{m}$. B: bone, S: scaphoid bone.

significantly increased above NC levels, indicating that the elbow joint was not affected by performance of this hand and wrist intensive repetitive task. For example, IL- $1 \alpha$ levels were $0.15 \pm 0.01$ (mean $\pm \mathrm{SEM}) \mathrm{pg} / \mu \mathrm{g}$ of total protein in HRHF compared to $0.22 \pm 0.04$ in NC; TNF- $\alpha$ was $0.35 \pm$ 0.05 in HRHF compared to $0.37 \pm 0.04$ in NC.

In summary, increased IL- $1 \alpha$ and TNF- $\alpha$ levels were present in the wrist joints (but not elbow joints) of HRHF rats compared to the other groups. These increases were attenuated by the ibuprofen treatment. IL-10 was highest in the TR rat wrist joints, but ibuprofen treatment in TR + IBU rats did not return IL-10 to baseline levels, suggesting that ibuprofen may be working through different mechanisms than IL-10 signaling cascades.
3.4. Histopathological Changes in the Distal Radial Articular Cartilage and Intracarpal Joints. We next investigated the distal radius articular cartilage for morphological changes occurring as a result of HRHF task performance and the effectiveness of secondary ibuprofen treatment in attenuating these changes. Figure 3(c) shows a low-power micrograph of HRHF radiocarpal joint with reduced safranin $\mathrm{O}$ staining in the radioscaphoid interterritorial articular cartilage, compared to NC (Figure 3(a)) and TR rats (Figure 3(b)). Higher-power micrographs show similar results (Figures 3(d)-3(i)). Reduced intraterritorial and pericellular proteoglycan staining (reduced safranin $\mathrm{O}$ staining) and structural changes (surface pannus, arrows) were observed in radial articular cartilages in untreated 
HRHF rats (Figures $3(\mathrm{f})$ and $3(\mathrm{~h})$ ), when compared to untreated TR (Figure 3(d)), TR + IBU (Figure 3(e)) or HRHF + IBU rats (Figures 3(g) and 3(i)). Cellular changes were also observed in the untreated HRHF rats, such as hypocellularity, which is indicative of cell loss (see areas indicated with $*$ in Figures 3(f) and 3(h)). Hypocellularity could also be observed in HRHF + IBU rats, although to a much lesser extent than in untreated HRHF rats (see Figure 3(i)). Finally, clustered chondrocytes were observed in untreated HRHF radial articular cartilage (see inset in Figures 3(f) and $3(\mathrm{~h})$ ). Chondrocyte clustering was also observed in HRHF + IBU rats, although, again to a much lesser extent than in untreated HRHF (see inset in Figures 3(g) and 3(i)). Clustering was not observed in TR, TR IBU or NC.

These changes were quantified using a modified Mankin scoring method, with results shown in Figure 4. As shown in Figure 4(a), each radiocarpal joint was assessed in four zones: zone 1 was the radial edge of articular cartilage, zone 2 was a radial area of articular cartilage ulnar to zone 1, zone 3 was an area of cartilage ulnar to zone 2, and zone 4 was the ulnar edge of the articular cartilage. All zones of articular cartilage were significantly different from control levels except zones 1 and 4 . Because these two zones were on the margins of the articular cartilage and may represent a transitional region of articular cartilage, only zones 2 and 3 were analyzed further.

The ANOVA for the overall histopathological scores of the distal radius articular cartilage demonstrated significant main effects for zone (zones 2 and 3) and group (Figure 4(a)), with the highest scores present in HRHF rats. The histopathological score in HRHF rats for zone 2 was slightly higher than that for zone 3 (Figure 4(a)). The averaged total Mankin score for both zones combined was also highest in HRHF rats compared to all groups (Figure 4(b)). We also assessed the subcomponents of the Mankin scoring system separately (Figures 4(c)-4(h)). Structural subscores in zone 2 ranged from $25 \%$ to $43 \%$ greater in the HRHF than in the other groups (mean score shown in Figure 4(c)). Thirty-three percent of the HRHF had more pronounced changes in the superficial layer (e.g., pannus) than in the other groups. None of the animals had structural changes beyond the superficial layers. Cellular scores were from 46 to $58 \%$ greater in the HRHF than those in the other groups, in both zones 2 and 3 (mean scores shown in Figures 4(e) and $4(\mathrm{f})$ ). All but one of the HRHF rats had chondrocyte clustering; $22 \%$ had regions of hypocellularity indicative of cell loss within the articular cartilage. Thirty-three percent of the HRHF + IBU had chondrocyte clustering (defined as hypercellularity with superficial clusters, and graded as 1), but none had hypocellular regions. Safranin O staining subscores were 79 to $257 \%$ greater (i.e., reduced proteoglycan staining) in the HRHF than in the other groups (mean scores shown in Figures 4(g) and 4(h)). All HRHF rats had reduced staining in the interterritorial matrix, $44 \%$ had staining only in the pericellular matrix, and one had a total absence of staining. Among the animals that did not perform the HRHF task, which includes NC, NC + IBU, TR and TR + IBU, only $38 \%$ had reduced staining in the interterritorial matrix.
Ibuprofen treatment attenuated the reduction in staining in HRHF rats (Figures $4(\mathrm{~g})$ and $4(\mathrm{~h}) ; P<.01$ ).

Distal radii and carpal bones probed for presence of cells with Terminal deoxynucleotidyl Transferase (TDT) mediated dUTP-biotin nick end-labeling (TUNEL). No TUNELstained cells were observed in the articular cartilages of radial bones in NC (Figures 5(a) and 5(g)) or TR rats (Figure 5(b)). Only a few TUNEL-labeled cells were seen in HRHF + IBU treated wrist articular cartilage (Figure 5(c)). In contrast, the radial bones of 5 of the 9-untreated HRHF rats showed scattered TUNEL-stained cells in the articular cartilages in zones 2 and 3 (zone 2 is shown in Figure 5(f); zone 3 is shown in Figures 5(d) (right side of photo) and 5(e)) and several TUNEL-stained cells at the edges of zone 4 (ulnar margin of radius; Figure 5(h)) and zone 1 (lateral or radial margin; left side of Figure 5(d)). This latter finding is interesting as these are sites of ligament attachments, such as the radiocarpal ligament shown in Figure 5(h). No TUNEL-stained cells were visible at the site of ligament attachment to the radius in the other groups (NC shown in Figure $5(\mathrm{~g})$ ). Most of the TUNEL-labeled cells observed in the HRHF radial articular cartilage were apoptotic in appearance (exhibited dark nuclear staining and cell condensation typically; Figures 5(i) and 5(j)). However, a few cells were also observed that were both swollen and had TUNEL staining in the cytoplasm as well as the nucleus, suggestive of necrosis (see inset of Figure 6(d)). In summary, modest morphological degenerative changes including reduced protoglycan staining and increased TUNEL-stained cells were present in radial articular cartilages of HRHF rats, changes attenuated by ibuprofen treatment.

Similar morphological changes were observed in the carpal bone articular cartilage located in intra-carpal joints. HRHF rats (Figure 6(b)) had reduced Safranin O and clustering (arrows) when visually compared to NC (picture not shown), TR (Figure 6(a)) and HRHF + IBU rats (Figure 6(c)). Again, ibuprofen treatment attenuated staining decreases observed in HRHF rats (compare Figures 6(b) to 6(c)). Epiphyseal plate changes were also observed in HRHF rats compared to the other groups. While Figure 3(c) shows patchy Safranin O staining in the epiphyseal plate of an HRHF rat, other HRHF rats had even greater losses, with a complete absence of Safranin O staining in the epiphyseal plates of a few (Figure 6(d)), compared to HRHF + IBU (Figure 6(e)), NC (Figure 3(a)) and TR (Figure 3(b)) rats.

3.5. Increased Serum Biomarkers of Collagen Degradation. To validate the histological findings, $\mathrm{C} 1,2 \mathrm{C}$, a serum biomarker of type I and II collagen degradation fragments was assessed. The HRHF had higher C1, 2C serum levels than NC, NC $+\mathrm{IBU}, \mathrm{TR}+\mathrm{IBU}$ and HRHF + IBU $(P=.01$ ANOVA; Figure $7(\mathrm{a})$ ), indicating greater collagen degradation in HRHF rats and its attenuation by IBU treatment. Serum was also evaluated for the CPII epitope, a serum biomarker reflective of type II collagen synthesis. Serum levels of CPII were significantly higher in HRHF rats compared to NC, TR and TR + IBU $(P=.006$ ANOVA; Figure $7(b))$. The ratio of collagen degradation to synthesis $(\mathrm{Cl}, 2 \mathrm{C} / \mathrm{CPII})$ was also higher in HRHF rats compared to NC, NC + IBU, and 


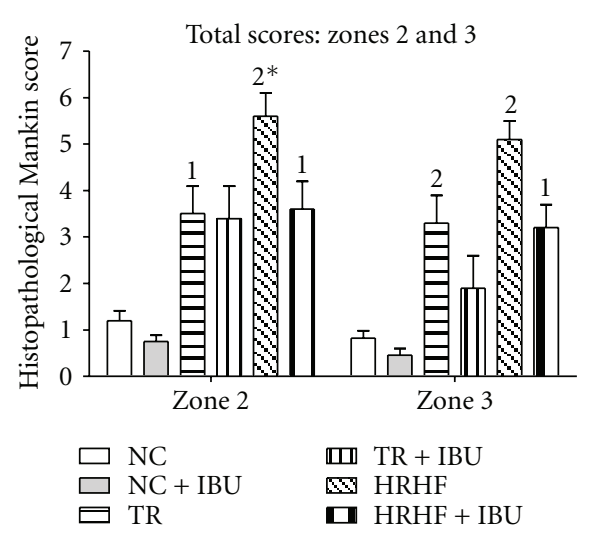

(a)

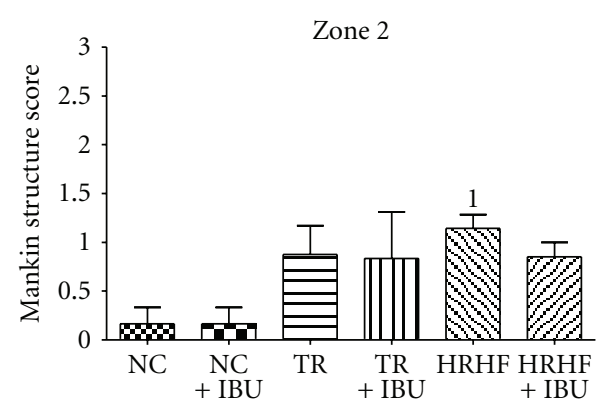

(c)

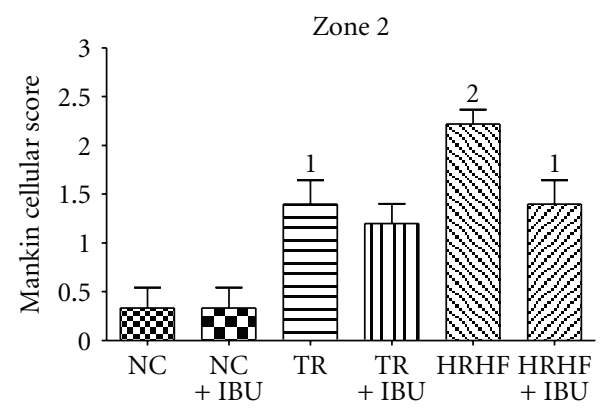

(e)

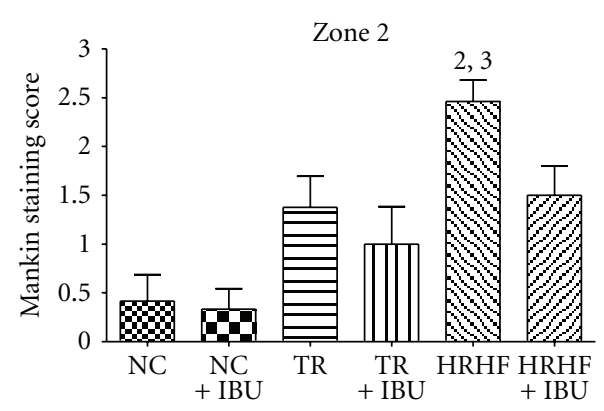

(g)

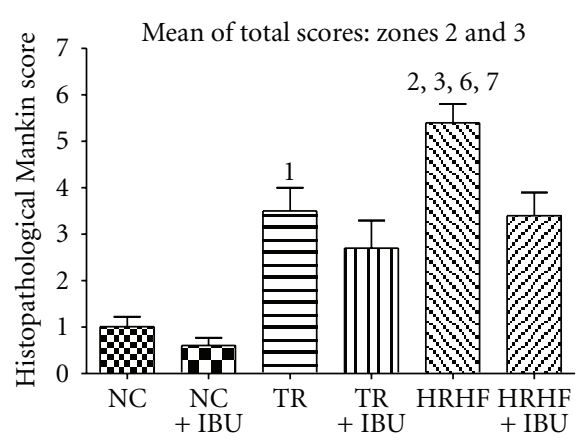

(b)

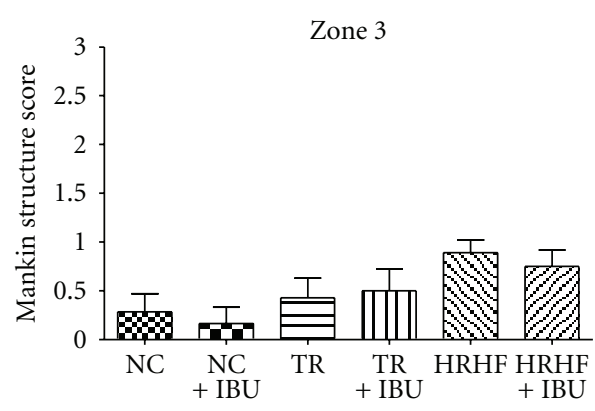

(d)

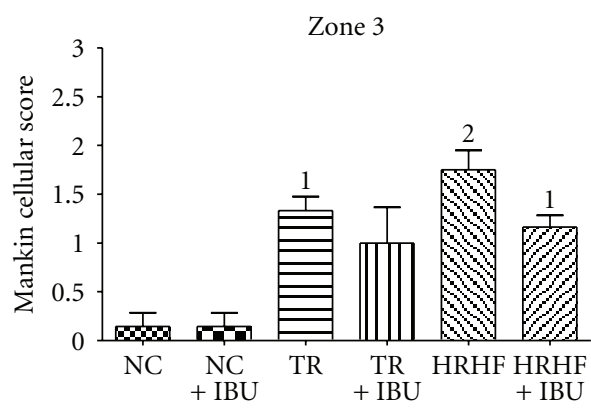

(f)

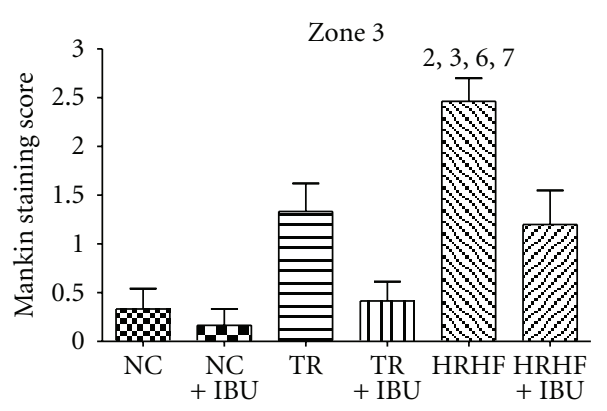

(h)

FIgURE 4: Histopathological Mankin scores shown for distal radius articular cartilage of the reach limb in NC (normal control), NC + IBU (normal controls receiving ibuprofen for 8 weeks), TR (trained controls), TR + IBU (trained controls receiving ibuprofen treatment in final 8 weeks), HRHF (rats performing high-repetition high-force task for 12 weeks), and HRHF + IBU (HRHF rats receiving ibuprofen treatment in final 8 weeks). (a) Total scores for zones 2 and 3 are presented separately by group. (b) Mean of total scores for both zones 2 and 3 . (c) Mankin score for structural changes in zone 2. (d) Mankin score for structural changes in zone 3. (e) Mankin score for cellular changes in zone 2. (f) Mankin score for cellular changes in zone 3. (g) Mankin score for staining changes in zone 2. (h) Mankin score for staining changes in zone 3. Abbreviations: 1 and $2=P<.05$ and $P<.01$, respectively, compared to NC. 3 and $4=P<.05$ and $P<.01$, respectively, compared to TR; 5 and $6=P<.05$ and $P<.01$, respectively, compared to TR + IBU; 7 and $8=P<.05$ and $P<.01$, respectively, compared to HRHF + IBU. $2^{*}=P<.001$ when comparing zones 2 and 3 (zone 2 is higher). Statistical results compared to NC + IBU are not presented as they were comparable to NC results. 


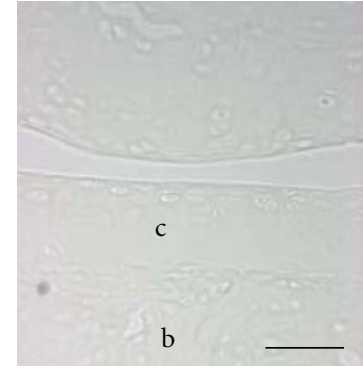

(a)

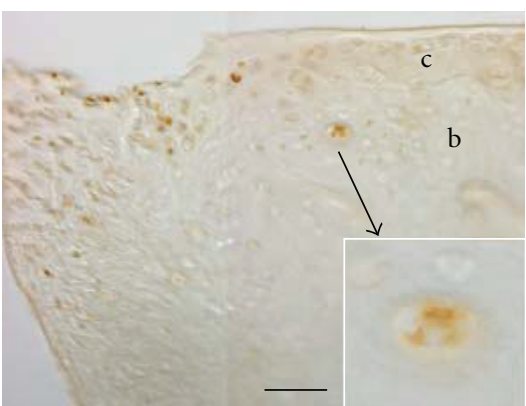

(d)

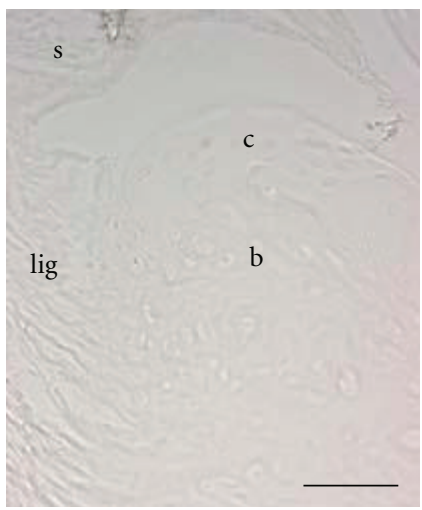

(g)

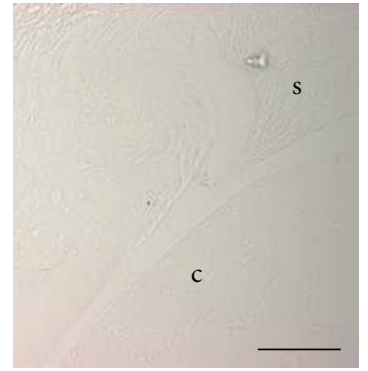

(b)

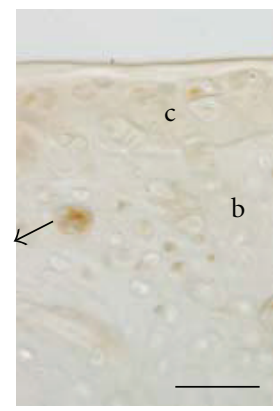

(e)

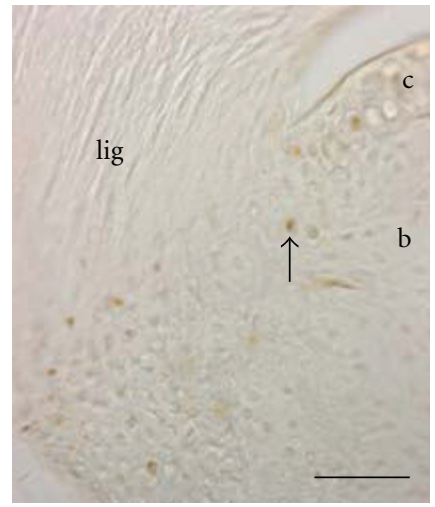

(h)

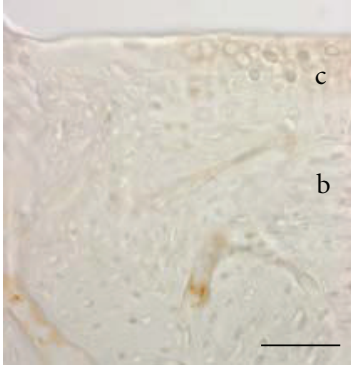

(c)

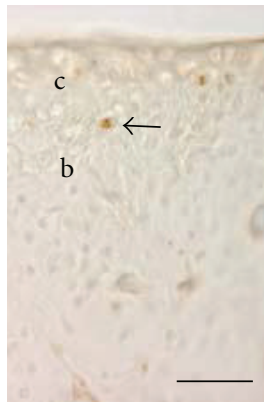

(f)

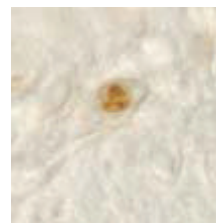

(i)

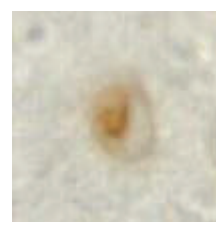

(j)

FIGURE 5: Distal radii and carpal bones stained with TUNEL (longitudinal sections of paraffin embedded radius and carpal bones). (a)-(f) Micrographs showing distal radius articular cartilage (indicated as c, for cartilage) in (a) NC (normal control), (b) TR (trained control), and (c) HRHF + IBU (performed the high-repetition high-force task for 12 weeks and received ibuprofen treatment daily in last 8 weeks), (d)-(e) HRHF rat (12 weeks HRHF rat with no treatment). (d) is a lower-power photo showing medial edge of radius and increased TUNEL stained cells at that edge as well as stained cells in adjacent articular cartilage. Inset shows higher-power image of indicated cell. (e) is a higher-power photo of same section shown in (d). (f) shows the radial articular cartilage from a different rat than shown in (d) and (e). (g) Image showing the lateral edge of the radius of an NC rat at the site of the attachment of the radiocarpal ligament. (h) Image showing the lateral edge of the radius of an HRHF rat at the site of the attachment of the radiocarpal ligament. (i) Higher-power photo of same cell as indicated in (f) with an arrow. (j) Higher-power photo of same cell as indicated in (h) with an arrow. Scale bars $=50 \mu \mathrm{m}$. b: bone, c: articular cartilage, lig: radiocarpal ligament, s: synovial ligament.

HRHF + IBU $(P=.02$ ANOVA; Figure $7(\mathrm{c}))$, indicating again greater collagen degradation in HRHF rats and its attenuation by IBU treatment. Significant post hoc findings are indicated in Figure 7.

\section{Discussion}

Numerous animal models have been employed to examine the effects of mechanical loading on joint structures; however, only a few studies have examined the effect of loading on upper extremity joint structures (e.g., $[43,44]$ ). Our model is nonsurgical and involves performance of a voluntary repetitive task to induce mechanical loading of forearm tissues. We selected the HRHF task as we have previously observed soft tissue and bone inflammation as well as degenerative changes, including bone pathological reorganization, using this task level $[26,32,38,45]$. The purpose of this study was to assess the potential of this 


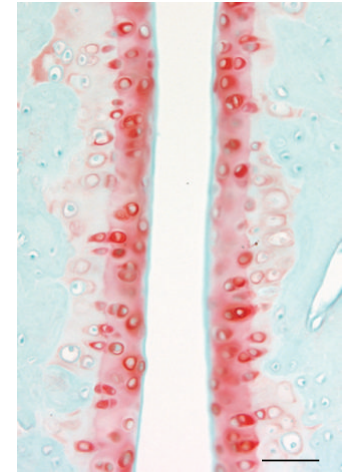

(a)

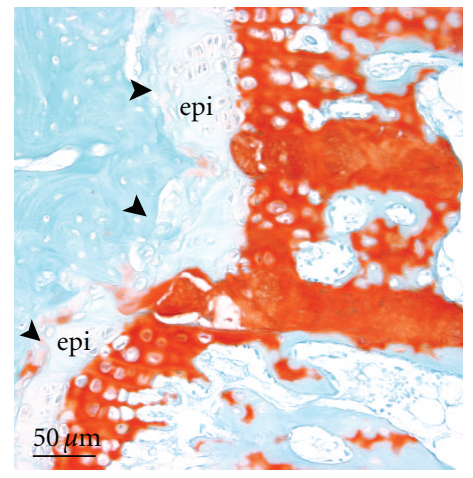

(d)

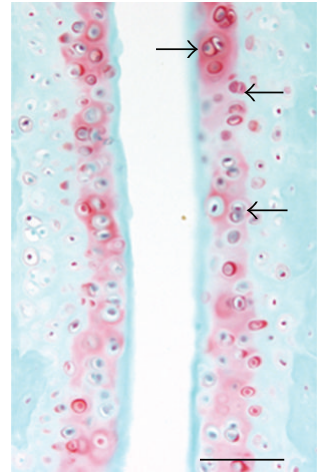

(b)

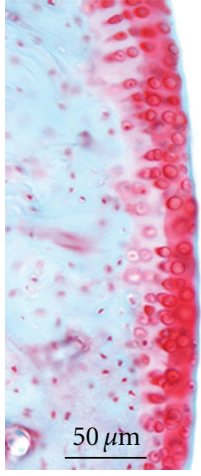

(c)

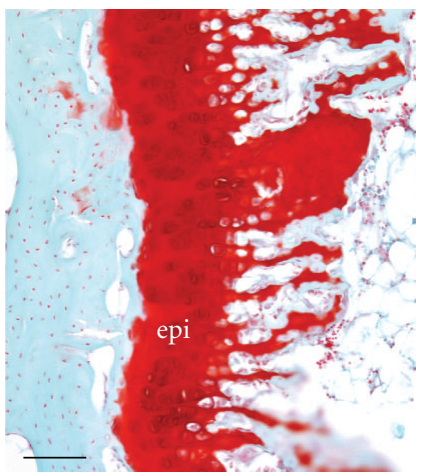

(e)

FIGURE 6: Carpal bone articular cartilages (intracarpal joints) and epiphyseal plates from longitudinal sections of paraffin embedded bone that were stained with safranin $\mathrm{O}$ and fast green. (a)-(c) Carpal bone articular cartilages in (a) TR (trained controls), (b) HRHF (rats performing high-repetition high-force task for 12 weeks), and (c) HRHF + IBU (HRHF rats receiving ibuprofen treatment in final 8 weeks). Reduced safranin O staining and a paucity of cells in lower cartilage layers are present in an HRHF rat, compared qualitatively to TR and HRHF + IBU. Chondrocytes that appear to be proliferating are present in the HRHF carpal articular cartilage (arrows). (d)-(e) Distal epiphyseal plate of the radius showing (d) an HRHF rat with a near absence of safranin O staining (arrowheads) and an irregularly shaped epiphyseal plate, as compared to (e) HRHF + IBU rat with an intensely stained and thicker epiphyseal plate. Scale bars $=50 \mu \mathrm{m}$. epi: epiphyseal plate.

high-demand hand-and-wrist intensive loading task in inducing inflammation and articular cartilage degeneration in radiocarpal and intracarpal joints, and to determine the effect of ibuprofen on these outcomes. After 12 weeks of performing the HRHF task, animals demonstrated evidence of joint inflammation (elevated inflammatory cytokine levels in distal radius, ulna and carpal bones, as well as increased $\mathrm{ED} 1+$ and COX2 + cells), the onset of joint degeneration (elevated modified Mankin histopathological scores in the distal radius articular cartilage, and increased TUNEL-labeled cells in the distal radius articular cartilage and attachment site of ligaments to the distal radius), increased C1, 2C (a biomarker of collagen degradation), and an increase in the ratio of collagen degradation to synthesis (C1, 2C/CPII). Eight weeks of ibuprofen administration reduced all of these changes, despite continued task performance and thus continued muscular loading during the 8 weeks treatment period of the HRHF + IBU group. This last finding indicates that the joint degenerative changes observed were a consequence of the inflammatory response induced by this high-repetition high-force task that was 12 weeks in duration.
We observed that 12 weeks of the HRHF task induced joint inflammation at the wrist, but not the elbow. This suggests that the cumulative loading during the 12 weeks task period of this high-repetition high-force handle pulling task affects distal structures more than proximal. This finding matches previous reports from our lab in which a highrepetition negligible-force (HRNF) task induced significantly higher numbers of ED1+ macrophages and osteoclasts in distal hand and wrist musculoskeletal tissues than in elbow and shoulder tissues [28, 30].

Concerning possible differential limb loading, IL-1 appeared more elevated in the distal bones/wrist joints of reach limbs than support limbs of TR and HRHF rats. Prior studies from our lab show that HRHF reach limbs had greater increases of several analytes than support limbs, including Substance P (a nociceptor neurotransmitter released after tissue injury/inflammation) and connective tissue growth factor (CTGF, a matricellular protein) in flexor digitorum tendons, and CTGF in the median nerve $[26,38]$. However, these studies also show that IL- $1 \beta$ in tendons, grip strength declines, and nerve conduction velocity declines were similar, 


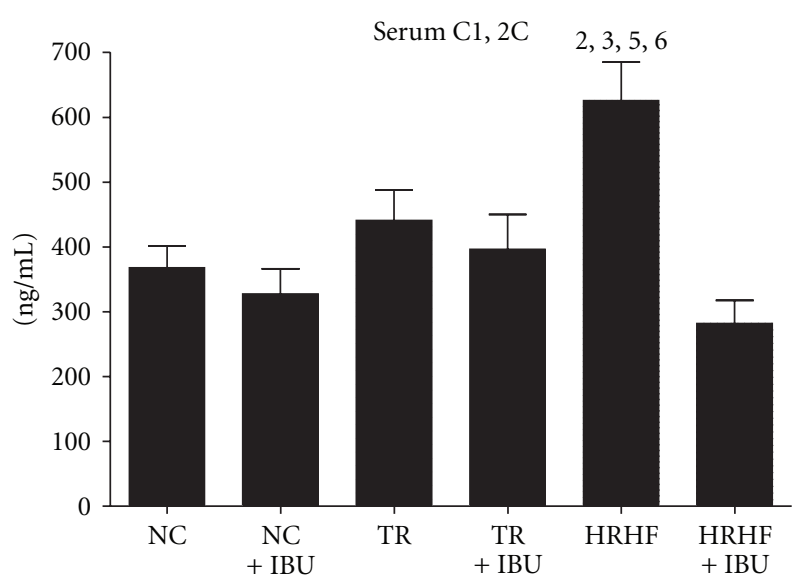

(a)

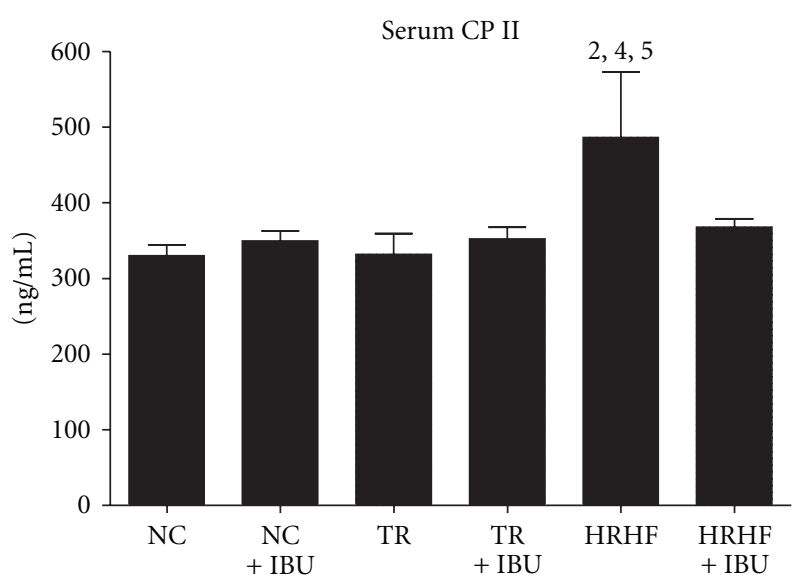

(b)

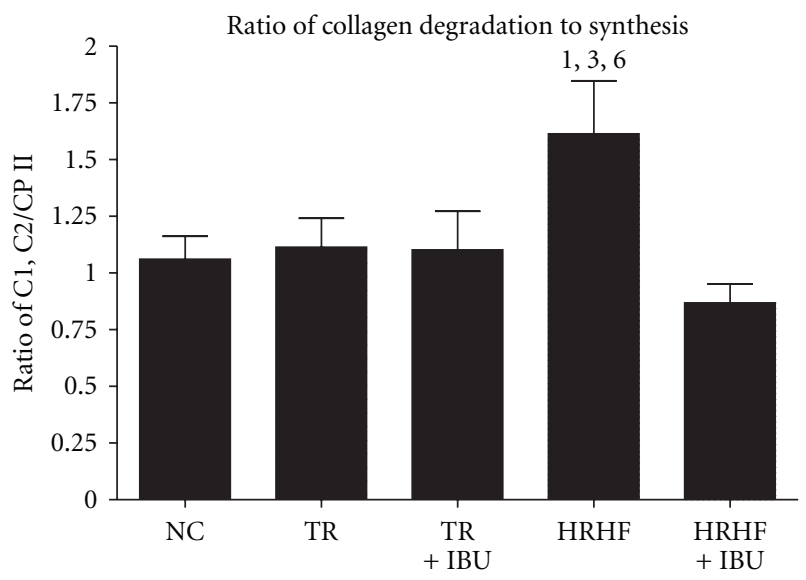

(c)

FIGURE 7: Serum concentrations of collagen degradation and synthesis markers, and the ratio of collagen degradation to synthesis in NC (normal control), NC + IBU (normal controls receiving ibuprofen for 8 weeks), TR (trained controls), TR + IBU (trained controls receiving ibuprofen treatment in final 8 weeks), HRHF (rats performing high-repetition high-force task for 12 weeks), and HRHF + IBU (HRHF rats receiving ibuprofen treatment in final 8 weeks). (a) C1, 2C, a marker of collagen Type I and II cleavage (degradation). (b) CPII, a marker of procollagen II C-propeptide synthesis. (c) Ratio of C1, C2 (degradation) to CPII (synthesis). $1=P<.05$ and $2=P<.01$ compared to NC; 3 $=P<.01$ compared to NC + IBU; $4=P<.05$ compared to TR; $5=P<.05$ compared to TR + IBU; $6=P<.01$ compared to HRHF + IBU.

bilaterally, in HRHF-trained and task rats [26, 38]. Since this HRHF task was a bilateral task, in which one limb reached and pulled the lever isometrically, and the other limb pushed against the chamber wall (see [38] for a depiction of this task), one has to be careful not to over interpret limb differences.

Our observed increases in cytokines in the distal bone and joint was not unexpected since degenerative joint osteoarthritis is associated with low-level inflammation within a joint $[6,46,47]$, and therefore, reduction of inflammation should attenuate degenerative processes. For example, increases of IL- $1 \alpha$, TNF- $\alpha$ and IL-10 have been reported in various tissues undergoing joint osteoarthritic degenerative changes (see [47] for a review), including articular cartilage [48-51], synovial membranes [46, 48] and subchondral bone [48]. In terms of the roles of these cytokines in joint degeneration, TNF- $\alpha$, for example, has been postulated to have a significant role in degradative cartilage changes associated with OA, since OA cartilage produces more TNF$\alpha$ and TNF alpha convertase enzyme mRNA than normal cartilage, and human articular chondrocytes from OA cartilage express higher amounts of p55 TNF- $\alpha$ receptors which could make OA cartilage particularly susceptible to TNF- $\alpha$ degradative stimuli [47]. In this current study, it was interesting that IL-10, an anti-inflammatory mediator, was highest in trained controls in this study, although it is unclear if this is a beneficial response to the training experience or a reaction to a simultaneous increase of IL- $1 \alpha$. A recent study assessed biochemical markers in synovium and synovial fluid between early- and late-stage knee osteoarthritis [52]. They found that 3 inflammatory mediators (IL- $1 \beta$, IL-6, and TNF- $\alpha$ ) in the synovium and synovial fluid were not significantly different between subsets. However, they also found that synovial fluid IL-15 concentrations were significantly greater in the 
early-stage than in the late-stage subset. The precise role of IL-15 in osteoarthritis is unclear, but it may be associated with proinflammatory and catabolic processes [52].

With regard to the ED1+ cells, our past studies show that activated macrophages and osteoclasts (both ED1+) are immunoreactive for several inflammatory cytokines, including IL- $1 \alpha$ and TNF- $\alpha[28,30,38]$. Since macrophages are known to secrete pro-inflammatory cytokines after injury, which, through autocrine regulation, induce further secretion of cytokines from this cell type, the increase of each with task performance and then decrease of each with ibuprofen treatment were [33] not unexpected in our study.

In concordance with human osteoarthritis, the HRHF task increased the number of cells in articular cartilage [53] and synovium [54-56] that were expressing COX-2, an enzyme associated with inflammation regulation. COX-2 expression is particularly elevated in synovium from earlystage joint degeneration $[55,56]$ and when chondrocytes experience high shear stresses [57]. We hypothesize that the HRHF task induced early joint degeneration after 12 weeks due to possible increased shear stresses on the articular cartilage, although this is hard to test in this in vivo model. We can only point out that the attenuation of COX-2 staining by ibuprofen, a nonselective COX inhibitor, combined with the attenuation of inflammatory cytokine levels, indicates that ibuprofen was at a therapeutic concentration.

We also observed that 12 weeks of performance of this HRHF task increased histopathological scores of radiocarpal joint degeneration. Our previous studies, using either this HRHF task or a more moderate HRNF task, have shown task-induced signs of injury and degeneration in forearm nerve, muscles, tendons and cortical bone [26-28, 30, 32, $38,45]$. In this study, the increased histopathological scores in untreated HRHF radiocarpal cartilage were primarily attributed to changes in staining and cellular subscores (e.g., decreased proteoglycan staining, surface irregularities, and increased clustering). Only three of 9 HRHF wrist joints had signs of definite structural changes (e.g., pannus), but 5 HRHF wrist joints showed increased TUNEL-labeled cells, indicative of the onset of task-induced chondrocyte apoptosis. The primary change was a loss of proteoglycan staining in radiocarpal cartilage as detected with Safranin O. Based on previous animal and human research, and based on the presence of TUNEL staining, if the HRHF task continued beyond 12 weeks, the degenerative changes would presumably worsen and propagate throughout the joint $[17,19,41,58,59]$. Since the histopathological scores were higher in the radial zone compared to the ulnar zone of radial articular cartilage, the radial region of this articular cartilage appears to be the initial site of joint degeneration in the reach limbs. This site is likely affected first because the forceful grasping in a pronated position increases the loading on the radial aspect of the articular cartilage. In the more radial zone, HRHF + IBU was not statistically different from HRHF. However, in a more ulnar zone (zone 3 ) these two groups were statistically different. We can only speculate that one reason for the zonal differences is that the radial zone began losing proteoglycans prior to week 4 (prior to when ibuprofen was administered) so the difference between the two groups was reduced. In contrast, zone 3 may have begun to lose proteoglycans closer to the start of ibuprofen treatment (or after), and therefore seems to demonstrate the differences between groups. Other nonsurgical joint degeneration models (e.g., undefined causes, spontaneous or knockout models) also report proteoglycan depletion and superficial cell clustering at 8 to 12 weeks and structural changes at 6 months of age $[18,58,59]$.

In addition to degenerative changes in the distal radius articular cartilage, HRHF carpal bones also showed signs of degenerative changes compared to the other groups. Unfortunately, the Mankin histopathological scoring system could not be used to score the articular cartilage morphology of rat carpal bones due to distinctive surface morphology differences in carpal bones compared to radial articular cartilage. However, degenerative carpal changes with HRHF were further supported by the presence of ED1+ mononucleated cells in subchondral carpal bone regions (see Figure 2(c)). Osteoclast progenitor cells are indicators of subchondral bone remodeling, a common feature of early joint degeneration [19].

The histopathological data indicative of cartilage degenerative changes were confirmed by increased serum $\mathrm{C} 1,2 \mathrm{C}$ (a marker of collagen types 1 and 2 degradation fragments produced by collagenase cleavage of type II collagen), increased CPII (a biomarker of procollagen II C-propeptide and thus collagen type II synthesis), and the increase in the ratio of $\mathrm{C} 1,2 \mathrm{C}$ to $\mathrm{CPII}$ (i.e., increased ratio of collagen degradation to synthesis). We have previously reported an early increase in serum osteocalcin (a biomarker of bone formation) by 6 weeks of performance of the HRHF task, which then decreased; and an increase in serum Trap5b (a biomarker of osteoclast activity and bone resorption) by 12 weeks [32]. The increase in serum osteocalcin was matched temporally by morphological and biochemical indicators of early bone adaptation changes, while the increase in serum Trap5b was matched temporally by morphological and biochemical indicators of bone resorption and pathology $[31,32]$. Our current results match studies of knee OA in patients in which cartilage turnover markers, for example, urinary CTX II (a biomarker of collagen type II degradation), increased as radiological and functional indicators of $\mathrm{OA}$ increased [60]. These results also match those in a study by Frisbe et al. [61] in which serum C1, 2C and CPII levels were increased in race horses with histological signs of early OA in mid-carpal joints compared to exercise-alone horses. In contrast to Frisbe's study, we did not observe increased CPII levels in the HRHF + IBU rats compared to resting controls (NC and TR rats), despite their continued performance of the HRHF task, suggesting that the increased CPII in our model is a consequence of the prolonged repetitive loadinginduced cytokine response rather than a direct consequence of daily activity.

Concerning the effectiveness of ibuprofen, we observed that 8 weeks of oral ibuprofen had both an anti-inflammatory and a chondroprotective effect on wrist joint structures in our model. Proinflammatory mediators (IL- $1 \alpha$ and TNF- $\alpha$ ) were reduced in HRHF + IBU rats compared to HRHF, and were at normal control levels. Ibuprofen 
also reduced IL-10, an anti-inflammatory and anticatabolic mediator, in HRHF + IBU limbs compared to HRHF reach limbs. IL-10 is stimulated by inflammatory mediators (e.g., by TNF- $\alpha$; [62]), upregulated in OA [49], and represents a feedback mechanism that inhibits inflammatory cytokines. Confusingly, though, IL-10 was not reduced to baseline levels in TR + IBU. Therefore, the influence of ibuprofen on IL10 concentrations is not straight-forward in our model. We do feel though that the long-term structural implications of suppressing IL-10 in HRHF limbs warrant further study.

Histopathological scores were also lower in HRHF + IBU than untreated HRHF rats, as was the presence of TUNEL-labeled cells, further supporting a chondroprotective effect of ibuprofen in our model. Our histopathological scoring results contradict findings by Jones et al. [33] with meloxicam, a nonsteroidal anti-inflammatory drug, in a surgically induced rat model for joint degeneration. In that study, meloxicam did not attenuate histopathological changes in articular cartilage. However, treatment with meloxicam prevented several other common characteristics of bone remodeling in early joint degeneration, including peri-articular trabecular bone loss and bone marrow lesion formation. The discrepancy in histopathological score results may be related to the use of different medications or the use of different joint degeneration models. Also, one limitation of this current study is that we did not assess periarticular trabecular bone changes using microcomputerized tomography (microCT), thus limiting the comparison of our findings to those by Jones.

Our observed reduction of serum $\mathrm{C} 1,2 \mathrm{C}$ levels and $\mathrm{C} 1$, 2C/CPII ratio in HRHF + IBU rats compared to untreated HRHF rats further supports the chondroprotective role of ibuprofen in our repetitive loading model, at least during this 12-week task period. This result is similar to patients with active knee OA, who when treated with ibuprofen for 4-6 weeks, had no increase in urinary CTX-II (a biomarker of collagen type II degradation) compared to patients with active knee OA treated with placebo only, in which urinary CTX-II was significantly increased [35]. Oral ibuprofen treatment for at least 2 weeks also decreases indexes of pain and functions significantly in patients with active knee OA, although the effectiveness lessens over time [34,63], However, these results contrast with those of Manicourt et al. [35], in which no reduction in urinary CTX-II or serum hyaluronan (HA; a marker of synovial inflammation) was observed in patients receiving a 4-week treatment of ibuprofen. Petersen et al. [37] also observed that ibuprofen treatment was not effective in attenuating cartilage turnover in OA patients in response to physical exercise. Perhaps these differences are due to the heterogeneity of OA in the human population [64].

In conclusion, 12 weeks of a voluntary high-repetition high-force reaching and handle pulling task represents a successful model for inducing wrist joint inflammation and early degenerative changes. Key proinflammatory and an anti-inflammatory cytokine levels, inflammatory cells, TUNEL-stained cells and histopathological scores of degeneration were elevated in joint tissues by 12 weeks of performance of this high-demand task. Each of these changes was attenuated by ibuprofen treatment, suggesting that such treatment is condroprotective, at least during the early phases of cumulative loading-induced inflammation and degeneration in hand and wrist joints.

\section{Conflict of Interests}

None of the authors have any conflicts of interest issues to declare.

\section{Acknowledgments}

This project was supported by Grant OH 03970 from CDCNIOSH to M. F. Barbe and Grant AR051212 from NIHNIAMS to A. E. Barr.

\section{References}

[1] Bureau of Labor Statistics, "Incidence of nonfatal occupational injuries and illnesses involving days away from work1 by occupation and selected parts of body affected by injury or illness," 2006, http://www.bls.gov/iif/oshwc/osh/case/ostb1889.pdf.

[2] Bureau of Labor Statistics, "Number of nonfatal occupational injuries and illnesses involving days away from work1 by occupation and selected parts of body affected by injury or illness," 2006, http://www.bls.gov/iif/oshwc/osh/case/ostb1666.pdf.

[3] Health Safety Executive, HSE, "Health Safety Executive, H.," 2006, http://www.hse.gov.uk/statistics/swi/swi0405.pdf.

[4] Health Safety Executive, HSE, 2007, http://www.hse.gov.uk/ statistics/lfs/lfs0506.pdf.

[5] Risquesprofessionals, "Prévention/études et dossiers/les troubles musculo—squelettiques," http://www.risquesprofessionnels.ameli.fr/fr/rubrique/prevention_etudes-dossiers_1.php.

[6] K. D. Brandt, P. Dieppe, and E. L. Radin, "Etiopathogenesis of osteoarthritis," Rheumatic Disease Clinics of North America, vol. 34, no. 3, pp. 531-559, 2008.

[7] R. P. Calfee, E. L. Leventhal, J. Wilkerson, D. C. Moore, E. Akelman, and J. J. Crisco, "Simulated radioscapholunate fusion alters carpal kinematics while preserving dart-thrower's motion," Journal of Hand Surgery, vol. 33, no. 4, pp. 503-510, 2008.

[8] J. A. Martin and J. A. Buckwalter, "Post-traumatic osteoarthritis: the role of stress induced chondrocyte damage," Biorheology, vol. 43, no. 3-4, pp. 517-521, 2006.

[9] B. Bernard, "Musculoskeletal disorders and workplace factors," NIOSH Report 97-141, National Institute for Occupational Safety and Health, Cincinatti, Ohio, USA, 1997.

[10] S. Solovieva, T. Vehmas, H. Riihimäki, K. Luoma, and P. Leino-Arjas, "Hand use and patterns of joint involvement in osteoarthritis. A comparison of female dentists and teachers," Rheumatology, vol. 44, no. 4, pp. 521-528, 2005.

[11] H. Ding, S. Solovieva, T. Vehmas, E. P. Takala, and P. LeinoArjas, "Hand osteoarthritis and pinch grip strength among middle-aged female dentists and teachers," Scandinavian Journal of Rheumatology, vol. 39, no. 1, pp. 84-87, 2010.

[12] L. Kalichman and G. Hernández-Molina, "Hand osteoarthritis: an epidemiological perspective," Seminars in Arthritis and Rheumatism, vol. 39, no. 6, pp. 465-476, 2010.

[13] K. L. Dominick, J. M. Jordan, J. B. Renner, and V. B. Kraus, "Relationship of radiographic and clinical variables to pinch and grip strength among individuals with osteoarthritis," Arthritis and Rheumatism, vol. 52, no. 5, pp. 1424-1430, 2005. 
[14] M. N. Spies-Dorgelo, D. A. W. M. van der Windt, H. E. van der Horst, A. P. A. Prins, and W. A. B. Stalman, "Hand and wrist problems in general practice-patient characteristics and factors related to symptom severity," Rheumatology, vol. 46, no. 11, pp. 1723-1728, 2007.

[15] A. M. Bendele, "Animal models of osteoarthritis in an era of molecular biology," Journal of Musculoskeletal Neuronal Interactions, vol. 2, no. 6, pp. 501-503, 2002.

[16] A. H. A. Abd, D. A. Hume, W. J. Halliday, and G. H. G. Davis, "Immunopathological investigations during the course of arthritis induced in rats by Streptococcus agalactiae," Medical Microbiology and Immunology, vol. 179, no. 1, pp. 13-23, 1990.

[17] K. Yamamoto, T. Shishido, T. Masaoka, and A. Imakiire, "Morphological studies on the ageing and osteoarthritis of the articular cartilage in C57 black mice," Journal of Orthopaedic Surgery, vol. 13, no. 1, pp. 8-18, 2005.

[18] E. N. Blaney Davidson, E. L. Vitters, P. M. van der Kraan, and W. B. van den Berg, "Expression of transforming growth factor- $\beta$ (TGF $\beta)$ and the TGF $\beta$ signalling molecule SMAD$2 \mathrm{P}$ in spontaneous and instability-induced osteoarthritis: role in cartilage degradation, chondrogenesis and osteophyte formation," Annals of the Rheumatic Diseases, vol. 65, no. 11, pp. 1414-1421, 2006.

[19] T. Hayami, M. Pickarski, Y. Zhuo, G. A. Wesolowski, G. A. Rodan, and L. T. Duong, "Characterization of articular cartilage and subchondral bone changes in the rat anterior cruciate ligament transection and meniscectomized models of osteoarthritis," Bone, vol. 38, no. 2, pp. 234-243, 2006.

[20] S. S. Glasson, T. J. Blanchet, and E. A. Morris, "The surgical destabilization of the medial meniscus (DMM) model of osteoarthritis in the $129 / \mathrm{SvEv}$ mouse," Osteoarthritis and Cartilage, vol. 15, no. 9, pp. 1061-1069, 2007.

[21] A. Kadri, T. Funck-Brentano, H. Lin et al., "Inhibition of bone resorption blunts osteoarthritis in mice with high bone remodelling," Annals of the Rheumatic Diseases, vol. 69, no. 8, pp. 1533-1538, 2010.

[22] M. B. Elliott, A. E. Barr, and M. F. Barbe, "Spinal substance $\mathrm{P}$ and neurokinin-1 increase with high repetition reaching," Neuroscience Letters, vol. 454, no. 1, pp. 33-37, 2009.

[23] M. B. Elliott, A. E. Barr, B. D. Clark, M. Amin, S. Amin, and M. F. Barbe, "High force reaching task induces widespread inflammation, increased spinal cord neurochemicals and neuropathic pain," Neuroscience, vol. 158, no. 2, pp. 922-931, 2009.

[24] M. B. Elliott, A. E. Barr, B. D. Clark, C. K. Wade, and M. F. Barbe, "Performance of a repetitive task by aged rats leads to median neuropathy and spinal cord inflammation with associated sensorimotor declines," Neuroscience, vol. 170, no. 3, pp. 929-941, 2010.

[25] M. B. Elliott, A. E. Barr, D. M. Kietrys, T. Al-Shatti, M. Amin, and M. F. Barbe, "Peripheral neuritis and increased spinal cord neurochemicals are induced in a model of repetitive motion injury with low force and repetition exposure," Brain Research, vol. 1218, no. C, pp. 103-113, 2008.

[26] B. D. Clark, T. A. Al-Shatti, A. E. Barr, M. Amin, and M. F. Barbe, "Performance of a high-repetition, high-force task induces carpal tunnel syndrome in rats," Journal of Orthopaedic and Sports Physical Therapy, vol. 34, no. 5, pp. 244-253, 2004.

[27] B. D. Clark, A. E. Barr, F. F. Safadi et al., "Median nerve trauma in a rat model of work-related musculoskeletal disorder," Journal of Neurotrauma, vol. 20, no. 7, pp. 681-695, 2003.
[28] M. F. Barbe, A. E. Barr, I. Gorzelany, M. Amin, J. P. Gaughan, and F. F. Safadi, "Chronic repetitive reaching and grasping results in decreased motor performance and widespread tissue responses in a rat model of MSD," Journal of Orthopaedic Research, vol. 21, no. 1, pp. 167-176, 2003.

[29] M. F. Barbe, M. B. Elliott, S. M. Abdelmagid et al., "Serum and tissue cytokines and chemokines increase with repetitive upper extremity tasks," Journal of Orthopaedic Research, vol. 26, no. 10, pp. 1320-1326, 2008.

[30] A. E. Barr, F. F. Safadi, I. Gorzelany, M. Amin, S. N. Popoff, and M. F. Barbe, "Repetitive, negligible force reaching in rats induces pathological overloading of upper extremity bones," Journal of Bone and Mineral Research, vol. 18, no. 11, pp. 20232032, 2003.

[31] S. Rani, M. F. Barbe, A. E. Barr, and J. Litivn, "Role of TNF alpha and PLF in bone remodeling in a rat model of repetitive reaching and grasping," Journal of Cellular Physiology, vol. 225, no. 1, pp. 152-167, 2010.

[32] S. Rani, M. F. Barbe, A. E. Barr, and J. Litvin, "Periostinlike-factor and Periostin in an animal model of work-related musculoskeletal disorder," Bone, vol. 44, no. 3, pp. 502-512, 2009.

[33] M. D. Jones, C. W. Tran, G. Li, W. P. Maksymowych, R. F. Zernicke, and M. R. Doschak, "In vivo microfocal computed tomography and micro-magnetic resonance imaging evaluation of antiresorptive and antiinflammatory drugs as preventive treatments of osteoarthritis in the rat," Arthritis and Rheumatism, vol. 62, no. 9, pp. 2726-2735, 2010.

[34] G. X. Qiu, S. N. Gao, G. Giacovelli, L. Rovati, and I. Setnikar, "Efficacy and safety of glucosamine sulfate versus ibuprofen in patients with knee osteoarthritis," Arzneimittel-Forschung, vol. 48, no. 5, pp. 469-474, 1998.

[35] D. H. Manicourt, M. Bevilacqua, V. Righini, J. P. Famaey, and J. P. Devogelaer, "Comparative effect of nimesulide and ibuprofen on the urinary levels of collagen type II Ctelopeptide degradation products and on the serum levels of hyaluronan and matrix metalloproteinases-3 and -13 in patients with flare-up of osteoarthritis," Drugs in R and D, vol. 6, no. 5, pp. 261-271, 2005.

[36] E. Gineyts, J. A. Mo, A. Ko et al., "Effects of ibuprofen on molecular markers of cartilage and synovium turnover in patients with knee osteoarthritis synovium turnover in patients with osteoarthritis," Annals of the Rheumatic Diseases, vol. 63, no. 7, pp. 857-861, 2004.

[37] S. G. Petersen, T. Saxne, D. Heinegard et al., "Glucosamine but not ibuprofen alters cartilage turnover in osteoarthritis patients in response to physical training," Osteoarthritis and Cartilage, vol. 18, no. 1, pp. 34-40, 2010.

[38] J. M. Fedorczyk, A. E. Barr, S. Rani et al., "Exposure-dependent increases in IL- $1 \beta$, substance P, CTGF, and tendinosis in flexor digitorum tendons with upper extremity repetitive strain injury," Journal of Orthopaedic Research, vol. 28, no. 3, pp. 298307,2010 .

[39] S. S. Adams, R. G. Bough, E. E. Cliffe et al., "Some aspects of the pharmacology, metabolism, and toxicology of ibuprofen. I. Pharmacology and metabolism," Rheumatology and physical medicine, vol. 10, pp. 10-26, 1970.

[40] J. O. Coq, F. Strata, M. Russier et al., "Impact of neonatal asphyxia and hind limb immobilization on musculoskeletal tissues and S1 map organization: implications for cerebral palsy," Experimental Neurology, vol. 210, no. 1, pp. 95-108, 2008. 
[41] J. A. van der Sluijs, R. G. T. Geesink, A. J. van der Linden, S. K. Bulstra, R. Kuyer, and J. Drukker, "The reliability of the Mankin score for osteoarthritis," Journal of Orthopaedic Research, vol. 10, no. 1, pp. 58-61, 1992.

[42] R. J. H. Custers, L. B. Creemers, A. J. Verbout, M. H. P. van Rijen, W. J. A. Dhert, and D. B. F. Saris, "Reliability, reproducibility and variability of the traditional Histologic/Histochemical Grading System vs the new OARSI Osteoarthritis Cartilage Histopathology Assessment System," Osteoarthritis and Cartilage, vol. 15, no. 11, pp. 1241-1248, 2007.

[43] M. R. van der Harst, J. DeGroot, G. H. Kiers, P. A. J. Brama, C. H. A. van de Lest, and P. R. van Weeren, "Biochemical analysis of the articular cartilage and subchondral and trabecular bone of the metacarpophalangeal joint of horses with early osteoarthritis," American Journal of Veterinary Research, vol. 66, no. 7, pp. 1238-1246, 2005.

[44] M. R. van der Harst, P. A. J. Brama, C. H. A. van de Lest, G. H. Kiers, J. DeGroot, and P. R. van Weeren, "An integral biochemical analysis of the main constituents of articular cartilage, subchondral and trabecular bone," Osteoarthritis and Cartilage, vol. 12, no. 9, pp. 752-761, 2004.

[45] S. Rani, M. F. Barbe, A. E. Barr, and J. Litvin, "Induction of periostin-like factor and periostin in forearm muscle, tendon, and nerve in an animal model of work-related musculoskeletal disorder," Journal of Histochemistry and Cytochemistry, vol. 57, no. 11, pp. 1061-1073, 2009.

[46] J. Bondeson, S. D. Wainwright, S. Lauder, N. Amos, and C. E. Hughes, "The role of synovial macrophages and macrophageproduced cytokines in driving aggrecanases, matrix metalloproteinases, and other destructive and inflammatory responses in osteoarthritis," Arthritis Research and Therapy, vol. 8, article R187, 2006.

[47] J. C. Fernandes, J. Martel-Pelletier, and J. P. Pelletier, "The role of cytokines in osteoarthritis pathophysiology," Biorheology, vol. 39, no. 1-2, pp. 237-246, 2002.

[48] H. Hulejová, V. Barešová, Z. Klézl, M. Polanská, M. Adam, and L. Šenolt, "Increased level of cytokines and matrix metalloproteinases in osteoarthritic subchondral bone," Cytokine, vol. 38, no. 3, pp. 151-156, 2007.

[49] F. Iannone, C. De Bari, F. Dell' Accio et al., "Interleukin-10 and interleukin-10 receptor in human osteoarthritic and healthy chondrocytes," Clinical and Experimental Rheumatology, vol. 19, no. 2, pp. 139-145, 2001.

[50] E. V. Tchetina, G. Squires, and A. R. Poole, "Increased type II collagen degradation and very early focal cartilage degeneration is associated with upregulation of chondrocyte differentiation related genes in early human articular cartilage lesions," Journal of Rheumatology, vol. 32, no. 5, pp. 876-886, 2005.

[51] J. Wang, P. Verdonk, D. Elewaut, E. M. Veys, and G. Verbruggen, "Homeostasis of the extracellular matrix of normal and osteoarthritic human articular cartilage chondrocytes in vitro," Osteoarthritis and Cartilage, vol. 11, no. 11, pp. 801809, 2003.

[52] C. R. Scanzello, E. Umoh, F. Pessler et al., "Local cytokine profiles in knee osteoarthritis: elevated synovial fluid interleukin15 differentiates early from end-stage disease," Osteoarthritis and Cartilage, vol. 17, no. 8, pp. 1040-1048, 2009.

[53] A. R. Amin, M. Attur, R. N. Patel et al., "Superinduction of cyclooxygenase-2 activity in human osteoarthritis-affected cartilage. Influence of nitric oxide," Journal of Clinical Investigation, vol. 99, no. 6, pp. 1231-1237, 1997.
[54] H. Seki, M. Fukuda, M. Iino, T. Takahashi, and N. Yoshioka, "Immunohistochemical localization of cyclooxygenase-1 and -2 in synovial tissues from patients with internal derangement or osteoarthritis of the temporomandibular joint," International Journal of Oral and Maxillofacial Surgery, vol. 33, no. 7, pp. 687-692, 2004.

[55] M. J. Benito, D. J. Veale, O. FitzGerald, W. B. van den Berg, and B. Bresnihan, "Synovial tissue inflammation in early and late osteoarthritis," Annals of the Rheumatic Diseases, vol. 64, no. 9, pp. 1263-1267, 2005.

[56] L. Ning, M. Ishijima, H. Kaneko et al., "Correlations between both the expression levels of inflammatory mediators and growth factor in medial perimeniscal synovial tissue and the severity of medial knee osteoarthritis," International Orthopaedics. In press.

[57] P. Wang, X. You, Y. Yan et al., "Cyclic mechanical stretch downregulates IL- $1 \beta$-induced COX-2 expression and $\mathrm{PGE}_{2}$ production in rheumatoid arthritis fibroblast-like synoviocytes," Connective Tissue Research. In press.

[58] K. Hu, L. Xu, L. Cao et al., "Pathogenesis of osteoarthritis-like changes in the joints of mice deficient in type IX collagen," Arthritis and Rheumatism, vol. 54, no. 9, pp. 2891-2900, 2006.

[59] C. M. Thomas, C. J. Fuller, C. E. Whittles, and M. Sharif, "Chondrocyte death by apoptosis is associated with cartilage matrix degradation," Osteoarthritis and Cartilage, vol. 15, no. 1, pp. 27-34, 2007.

[60] P. Garnero, N. Charni, F. Juillet, T. Conrozier, and E. Vignon, "Increased urinary type II collagen helical and C telopeptide levels are independently associated with a rapidly destructive hip osteoarthritis," Annals of the Rheumatic Diseases, vol. 65, no. 12, pp. 1639-1644, 2006.

[61] D. D. Frisbie, F. Al-Sobayil, R. C. Billinghurst, C. E. Kawcak, and C. W. McIlwraith, "Changes in synovial fluid and serum biomarkers with exercise and early osteoarthritis in horses," Osteoarthritis and Cartilage, vol. 16, no. 10, pp. 1196-1204, 2008.

[62] C. Ritchlin and S. A. Haas-Smith, "Expression of interleukin 10 mRNA and protein by synovial fibroblastoid cells," Journal of Rheumatology, vol. 28, no. 4, pp. 698-705, 2001.

[63] P. Muller and B. Simon, "Effects of ibuprofen lysinate and acetylsalicylic acid on gastric and duodenal mucosa. Randomized single-blind placebo-controlled endoscopic study in healthy volunteers," Arzneimittel-Forschung, vol. 44, no. 7, pp. 840-843, 1994.

[64] J. B. Driban, M. R. Sitler, M. F. Barbe, and E. Balasubramanian, "Is osteoarthritis a heterogeneous disease that can be stratified into subsets?" Clinical Rheumatology, vol. 29, no. 2, pp. 123$131,2010$. 


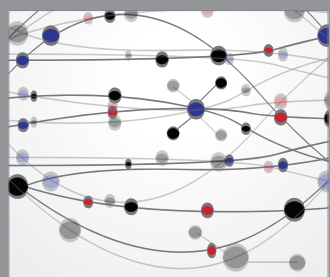

The Scientific World Journal
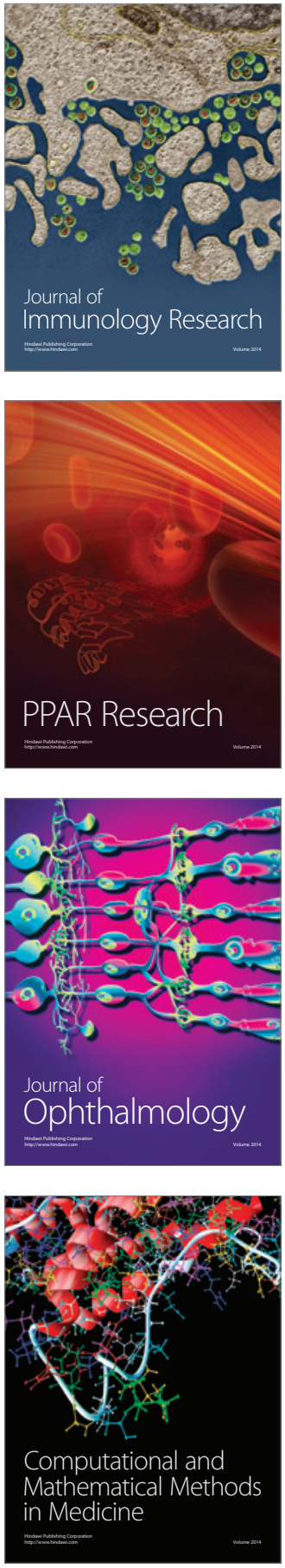

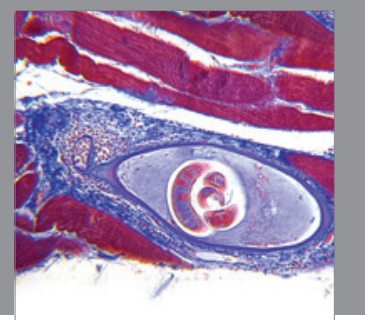

Gastroenterology

Research and Practice
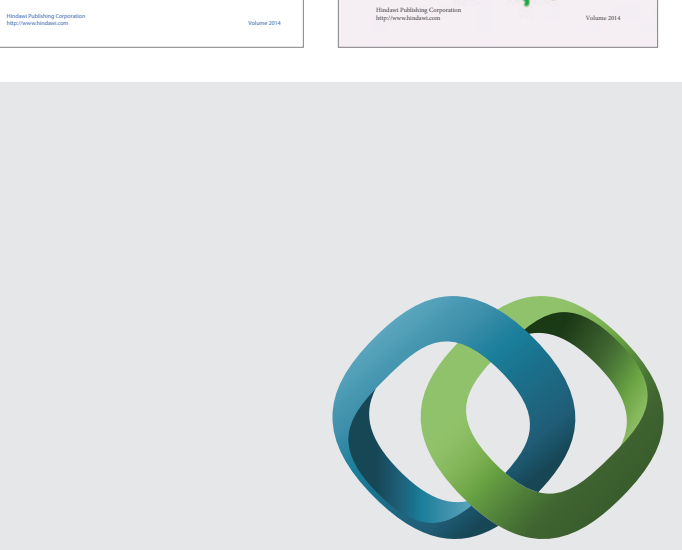

\section{Hindawi}

Submit your manuscripts at

http://www.hindawi.com
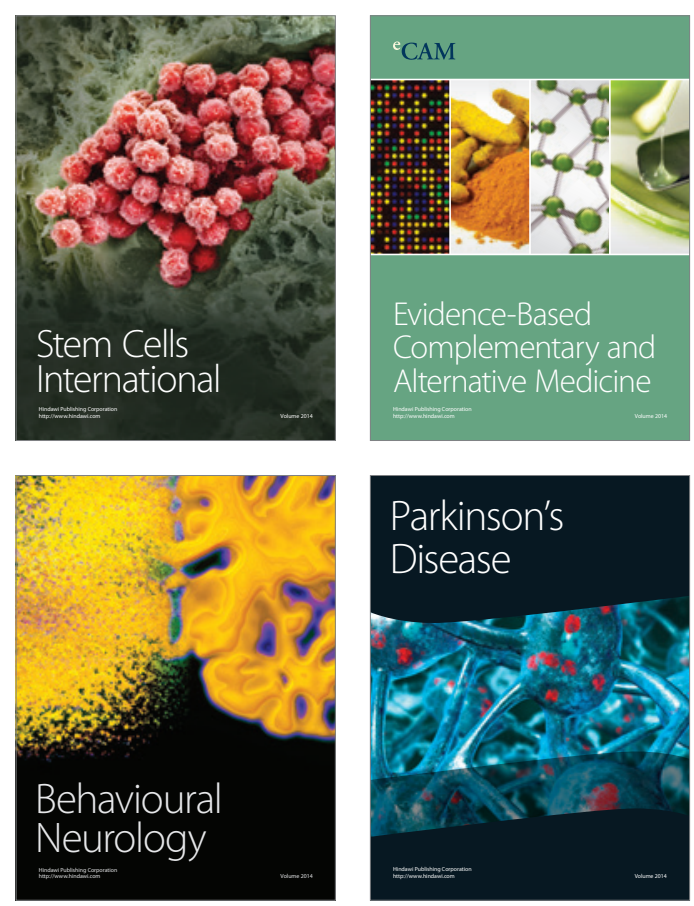

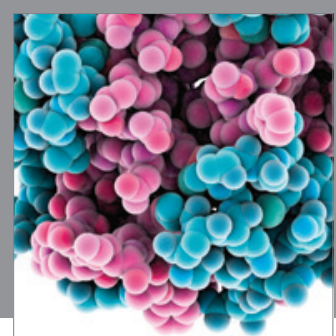

Journal of
Diabetes Research

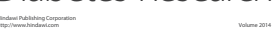

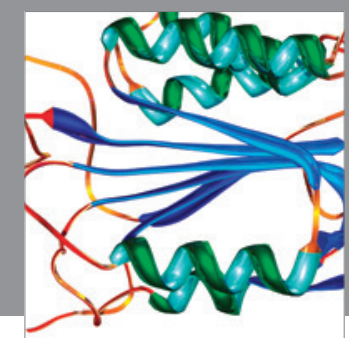

Disease Markers
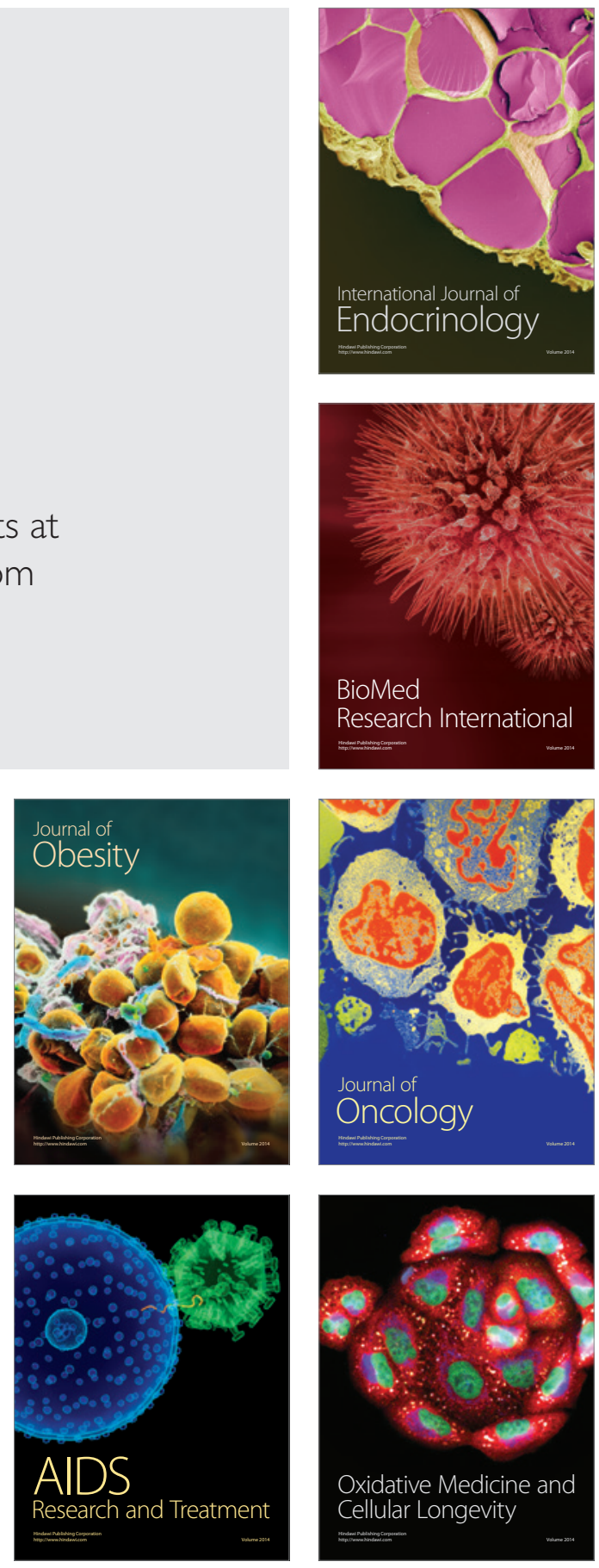